\title{
Network analysis of attitudes towards immigrants in Asia
}

\author{
Rachael Kei Kawasaki* and Yuichi Ikeda
}

\author{
* Correspondence: kawasaki.kei. \\ 52e@st.kyoto-u.ac.jp \\ Graduate School of Advanced \\ Integrated Studies in Human \\ Survivability, Kyoto University, 1 \\ Nakaadachi-cho, Yoshida, Sakyo-ku, \\ Kyoto 606-8306, Japan
}

\begin{abstract}
This study models cross-national attitudes towards immigrants in East and Southeast Asia as a signed and weighted bipartite network of countries and evaluative reactions to a variety of political issues, or determinants. This network is then projected into two one-mode networks, one of countries and one of determinants, and community detection methods are applied. The paper aims to fill two deficiencies in the current research on attitudes towards immigrants: 1) the lack of cross-national studies in Asia, a region where migration is growing, and 2) the tendency of researchers to treat determinants as uncorrelated, despite the interdependent nature of evaluative reactions. The results show that the nine countries in the sample are a cohesive clique, showing greater similarities than differences in the determinants of their attitudes. A blockmodeling approach was employed to identify eight determinants in attitudes towards immigrants, namely views on independence and social dependencies, group identities, absolute or relative moral orientation, attitudes towards democracy, science and technology, prejudice and stigma, and two determinants related to religion. However, the findings of this survey yielded some surprising results when compared with the literature review. First, education was not found to be a significant determinants of attitudes towards immigrants, despite its strong and consistent predictive power in European models. Second, prejudice appears to be mediated in part by religion, especially in religious identification and belief in God. Group identity and prejudice also appear to be related, though only weakly. Finally, anxiety appears in clusters related to social norms, suggesting that fears regarding immigrants relates closely to expectations of others' behavior.
\end{abstract}

Keywords: Migration, Attitudes, Bipartite network, Blockmodeling

\section{Introduction}

The past decade has seen migration become a more controversial issue in myriad countries, with stalwart supporters and detractors. Not only does the level of immigration spark impassioned debate, but what happens after immigrants arrive and settle in a country is equally salient. Of particular interest to policymakers and publics is

(c) The Author(s). 2020 Open Access This article is licensed under a Creative Commons Attribution 4.0 International License, which permits use, sharing, adaptation, distribution and reproduction in any medium or format, as long as you give appropriate credit to the original author(s) and the source, provide a link to the Creative Commons licence, and indicate if changes were made. The images or other third party material in this article are included in the article's Creative Commons licence, unless indicated otherwise in a credit line to the material. If material is not included in the article's Creative Commons licence and your intended use is not permitted by statutory regulation or exceeds the permitted use, you will need to obtain permission directly from the copyright holder. To view a copy of this licence, visit http://creativecommons.org/licenses/by/4.0/. 
whether immigrants become an accepted part of society, or if they and their children remain at the margins of society.

Attitudes towards immigrants and immigration (ATII) ${ }^{1}$ are definitional aspect the integration of immigrants into a society. While integration is a process that includes multiple dimensions, such as labor market inclusion, civil participation, etc., it can also be defined simply as becoming an accepted part of society (Penninx 2007). A large proportion of negative attitudes towards immigrants amongst a country's citizenry by definition shows that immigrants and immigrant groups have not become a fully acceptable part of society. Moreover, negative attitudes have practical effects on other aspects of immigrants' integration. Areas where the public has more negative attitudes have been found to have greater housing discrimination against immigrants (Carlsson and Eriksson 2017). Immigrants tend to have lower life satisfaction (Knabe et al. 2013) and greater strains on mental and physical health (Agudelo-Suárez et al. 2009). Investigating why migration is acceptable to some and anathema to others is, thus, of critical importance in ensuring the well-being of all residents, creating a more cohesive society, and guiding policymakers and NGOs in developing more effective and amenable immigration and integration policies.

Given the increasing salience of how attitudes towards immigrants are formed, the current research on the determinants of attitudes towards immigrants has begun to reveal its shortcomings. First, research, especially cross-national research, has been largely limited to European countries and Settler countries, as Table 1 shows. Settler countries here refer to Australia, Canada, New Zealand, and the United States, all former colonies of the United Kingdom which experienced large scale immigration in the eighteenth and nineteenth century.

There are a several reasons for this overemphasis of European and Settler countries, including an unequal distribution of research resources and capacity in the Global North (Castles 2010) and greater availability and depth of data in European and Settler countries (UN DESA 2017). However, as the destination and origins of migrants change, this lack of understanding of how different countries react to the inclusion of immigrants into a society leads to a poorer ability to make and communicate effective immigration and integration policy. Asia recently overtook Europe as the region hosting the greatest number of migrants in the world and experienced the largest growth in migrant stock in the period between 2000 and 2017 (UN DESA 2017). The current research has thus far failed to reflect these changing conditions.

The overemphasis of Western countries has led to methodological problems and assumptions that compromise the generalizability of research findings and which limit its applicability to Asian countries. These oversights include assumptions of liberal democracies, higher development levels, European and Settler country cultural norms related to ingroups and outgroups, and so on. The effect of this overemphasis is that models and findings are limited to only one region and lose accuracy as the survey expands to other regions (Mayda 2006). Variables that are not relevant to European and Settler countries are overlooked. The scope of the previous research limits the amount of research into structural determinants of ATII, e.g. development level, government corruption, inequality, etc. Without the inclusion of a more diverse group of countries,

${ }^{1}$ See Ceobanu and Escandell (2010) for a discussion of this term. 
Table 1 Cross-national ATIl studies using survey data: locations

\begin{tabular}{|c|c|c|c|c|}
\hline Location & $\begin{array}{l}\text { Max. } \\
\text { number of } \\
\text { countries }\end{array}$ & $\begin{array}{l}\text { Max. number of non- } \\
\text { European, non-Settler } \\
\text { countries }\end{array}$ & Data set & Study \\
\hline \multirow[t]{10}{*}{ Europe } & 17 & 0 & $\begin{array}{l}\text { European Social } \\
\text { Survey (ESS) }\end{array}$ & $\begin{array}{l}\text { Meuleman et } \\
\text { al. (2009) }\end{array}$ \\
\hline & 21 & 0 & ESS & $\begin{array}{l}\text { Gorodzeisky } \\
\text { and Semyonov } \\
\text { (2009) }\end{array}$ \\
\hline & 22 & 0 & ESS & $\begin{array}{l}\text { Card et al. } \\
(2005)\end{array}$ \\
\hline & 22 & 0 & ESS & $\begin{array}{l}\text { Hainmueller } \\
\text { and Hiscox } \\
\text { (2007) }\end{array}$ \\
\hline & 26 & 0 & ESS & $\begin{array}{l}\text { Malchow-Møller } \\
\text { et al. (2009) }\end{array}$ \\
\hline & 12 & 0 & Eurobarometer & $\begin{array}{l}\text { Gang et al. } \\
\text { (2013) }\end{array}$ \\
\hline & 15 & 0 & Eurobarometer & Lahav (2004) \\
\hline & 17 & 0 & Eurobarometer & $\begin{array}{l}\text { Coenders et al. } \\
\text { (2005) }\end{array}$ \\
\hline & 15 & 0 & Eurobarometer & $\begin{array}{l}\text { Kessler and } \\
\text { Freeman (2005) }\end{array}$ \\
\hline & 12 & 0 & Eurobarometer & $\begin{array}{l}\text { Semyonov et al. } \\
\text { (2006) }\end{array}$ \\
\hline \multirow[t]{2}{*}{$\begin{array}{l}\text { North America, South } \\
\text { America }\end{array}$} & 17 & 17 & Latin Barometer & $\begin{array}{l}\text { Lawrence } \\
(2011)\end{array}$ \\
\hline & 10 & 10 & $\begin{array}{l}\text { Latin American } \\
\text { Public Opinion } \\
\text { Project (LAPOP) }\end{array}$ & $\begin{array}{l}\text { Meseguer and } \\
\text { Kemmerling } \\
\text { (2016) }\end{array}$ \\
\hline $\begin{array}{l}\text { Africa, Asia, Europe, North } \\
\text { America, South America }\end{array}$ & 31 & 8 & $\begin{array}{l}\text { International Social } \\
\text { Survey Programme } \\
\text { (ISSP) }\end{array}$ & $\begin{array}{l}\text { Facchini and } \\
\text { Mayda (2008) }\end{array}$ \\
\hline \multirow{2}{*}{$\begin{array}{l}\text { Africa, Asia, Europe, North } \\
\text { America, Oceania, South } \\
\text { America }\end{array}$} & 53 & 40 & $\begin{array}{l}\text { World Values Survey } \\
\text { (WVS) }\end{array}$ & $\begin{array}{l}\text { Cooray et al. } \\
\text { (2018) }\end{array}$ \\
\hline & 66 & 22 & ISSP; WVS & Mayda (2006) \\
\hline
\end{tabular}

identifying which determinants are truly generalizable and which are country-specific as well as how macro-level factors affect ATII is difficult.

Finally, the previous research on ATII assumes that variables are uncorrelated, despite the evidence in the psychological literature that attitudes form in a network structure. Putting attitudes in a bipartite network structure not only coheres more closely with theories related to human cognition (Fazio 1986; Fazio 1990; Monroe and Read 2008; van Overwalle and Siebler 2005), it also allow us to identify how countries differ or are similar in their determinants. Thus, how ATII form and its determinants is a natural but underexplored area for the application of network science techniques.

This study will identify and analyze the determinants of attitudes towards immigrants in nine East and Southeast Asian countries in a network structure, showing not only which determinants are salient in this critical and understudied region of migration but also the relationships between these determinants. Given that little of the previous cross-national research has been conducted in this region, this study aims to recognize new and understudied trends and variables, with the hope of inspiring greater research 
into these areas. Using data from the sixth wave of the World Values Survey, countries and variables will first be arranged in a bipartite network, before being projected into one-mode networks. Community detection within the country network will allow for a greater understanding of how countries are similar or unique in their determinants. The variable network is then organized in a blockmodel, allowing for dimensionality reduction. The most centrally located node, defined as the node with the largest node strength in the block, is then selected as the representative variable for the block. This method allows us to identify eight relevant determinants of attitudes towards immigrants: independent, group identity, absolute or relative moral orientation, democracy, science and technology, prejudice and stigma, and two determinants related to religion. Moreover, this network structure allows us to explore the relationships amongst determinants.

This paper is organized as follows: first, the related work on attitudes towards immigrants will be reviewed. Next, the data and methodology used in the study will be described. The results of this methodology and its implications will be discussed, before concluding.

\section{Related works}

As mentioned above, attitudes form based on a number of considerations including other attitudes. Attitudes towards one object rely on relevant evaluations of other objects, the accessibility of these evaluations, the respondent's affect, mood, and emotion as well as new information, the source of this information, and the respondent's relationship with the source (Crano and Prislin 2006). People can also hold contradicting evaluations of the same object, either through implicit and explicit evaluations that are more or less accessible depending on the situation (Wilson et al. 2000) or through invalidating previous evaluations (Petty et al. 2006). The state of holding conflicting evaluations towards the same object is often referred to as "cognitive dissonance" (Festinger 1957). Which evaluation is expressed depends on the relative strength of each evaluation as well as the motivation of the individual, external conditions, and so on (Wilson et al. 2000). As people try to avoid the discomfort of cognitive dissonance, they may seek out and retain information that supports their view to the detriment of opposing facts (Frey 1986; Brannon et al. 2007; Hart et al. 2009). Thus, attitudes depend on other attitudes, the strength of these attitudes and evaluations, the social network of the respondent, mood, and so on. While some of these factors are outside the scope of the widely available survey data, a respondents' evaluation of other political and social aspects are included in the WVS.

Because of the dependencies between evaluations in creating attitudes, network science has been employed to model the formation of attitudes towards a variety of public opinion issues. Connectionist accounts of attitudes posit that political attitudes, like attitudes towards immigrants and immigration, are formed through the influence of relevant and related beliefs, which are in turn reliant on other attitudes and evaluations (Monroe and Read 2008; van Overwalle and Siebler 2005). This theory has several advantages over other theories of attitude formation and change. First, it produces a model which mirrors the human brain and which allows for learning through the adoption of new pieces of information, represented by nodes, and through the forging of new pathways between nodes. Through the attribute of attitude strength, this network approach can help explain 
why some attitudes are weak and subject to change, while stronger, more central attitudes remain stable throughout life (van Overwalle and Seiber 2005). Moreover, modeling attitudes as network can help explain how forming new attitudes or accessing old ones can be near automatic. As van Overwalle and Seiber state, "these systems do not need a central executive, which eliminates the requirement of central and deliberative processing of attitude information" (van Overwalle and Seiber 2005: 232). This decentralized mechanism also encourages maintaining consistency amongst attitudes, an impulse which can be observed when respondents are asked about multiple attitudes (Dalege et al. 2016). Finally, unlike a latent factor approach, using a network structure allows for observed variables to have causal influence on the dependent variable, rather than crediting all influence to an unobserved factor (Dalege et al. 2016).

One such model is the Causal Attitude Network model (CAN), an empirical application of interrelated evaluative reactions towards an object (Dalege et al. 2016). The network is created by regressing each node, in this case an evaluative reaction towards the object, against another. The parameters of this logistic regression than become the edge weights of the ties between the nodes. This model has been applied to post-national citizenship identities (whether one identifies more strongly as a citizen of the world or of a transnational, supranational identity than as a citizen of their country) using data from 27 European OECD member countries (Schlicht-Schmälzle et al. 2018). While differences between younger and older respondents, rural and urban respondents, etc., are found through a series of Network Comparison Tests, the model does not show differences and similarities amongst countries.

Methodological problems notwithstanding, a wealth of research has been conducted in the determinants of attitudes towards immigrants, and cross-national studies have identified a variety of consistent determinants. Table 2 summarizes and categorizes these factors. Determinants are split into individual-level (both attitudinal and non-

Table 2 Summary of determinants of ATII

\begin{tabular}{|c|c|c|c|}
\hline Group & & Factors & Study \\
\hline \multirow[t]{12}{*}{ Individual } & \multirow[t]{5}{*}{ Non-attitudinal } & Education & Hainmueller and Hiscox (2007) \\
\hline & & Age & Gorodzeisky and Semyonov (2009) \\
\hline & & Sex & Gorodzeisky and Semyonov (2009) \\
\hline & & Rural/urban residence & Gorodzeisky and Semyonov (2009) \\
\hline & & Economic self-interest & Mayda (2006) \\
\hline & \multirow[t]{7}{*}{ Attitudinal } & Value of cultural homogeneity & Sides and Citrin (2007) \\
\hline & & Social Dominance Orientation & Newman et al. (2014) \\
\hline & & Satisfaction with democracy in their country & Kunovich (2009) \\
\hline & & Political orientation & Kunovich (2009) \\
\hline & & Issue salience & Dennison and Geddes (2019) \\
\hline & & Ethnocentrism/prejudice & Burns and Gimpel (2000) \\
\hline & & Religion & Scheepers et al. (2002) \\
\hline \multirow[t]{2}{*}{ Group } & & Group contact & Schlueter and Wagner (2008) \\
\hline & & National Identity & Mummendey et al. (2001) \\
\hline \multirow[t]{3}{*}{ Macro } & & National economic conditions & Coenders et al. (2008) \\
\hline & & (Perceptions) of local immigrant population & Hjerm (2007) \\
\hline & & Political system & Coenders and Scheepers (2003) \\
\hline
\end{tabular}


attitudinal), group-level determinants, and macro-level factors. Macro-level factors are currently outside the purview of this study, but are included in Table 2.

\section{Individual-level: non-attitudinal}

In debates about immigration, pundits and policymakers often emphasize the individual economic situation of respondents, stating that negative attitudes are motivated by a decrease in economic well-fare of respondents, either through a decrease in wages (Mayda 2006; Coenders et al. 2008; Jackson et al. 2001; Kehrberg 2007; Semyonov et al. 2006; Semyonov et al. 2008; Wilkes et al. 2007) or through an increase in fiscal burden (Facchini and Mayda 2009; Campbell et al. 2006; Dustman and Preston 2007). However, there is little empirical evidence for this effect, so much so that Hainmueller and Hopkins refer to this theory as "a zombie theory" (2014, pp. 241). However, there is some evidence that people's perception of how the national economy as a whole is faring do affect ATII (Coenders et al. 2008; Lahav 2004; Semyonov et al. 2008).

Education, on the other hand has been found to be significant in most studies on ATII conducted in European and Settler countries (Lancee and Sarrasin 2015; Freeman et al. 2013). As level of education increases, especially to or past the tertiary level, support for immigration increases and anti-immigrant sentiment decreases. However, its effects are not consistent across countries. While the effect of education on attitudes is remarkably strong in Western Europe, this effect weakens in Central and Eastern Europe, suggesting that the effect of education is mediated by other factors (Coenders and Scheepers 2003; Hello et al. 2002). Studies outside of Europe also find that education has little predictive power; Meseguer and Kemmerling's (2016) study in 10 Latin American countries of varying development levels, finds no effect of education on attitudes once other variables are included.

\section{Individual-level: attitudinal factors}

Individual attitudinal factors are the focus of the pre-selection method for identifying relevant variables. These factors especially benefit from being put into a network structure, as attitudes form and are shaped by relevant attitudes towards other political or social issues. Prejudice against other ethnicities has been found overwhelmingly to be linked towards anti-immigrant prejudice, though a central question remains as to whether prejudice against immigrants is origin-blind (Citrin et al. 1997; Sniderman et al. 2000; Kinder and Kam 2010) or if people differentiate their attitudes towards immigrants based on country of origin, ethnicity, and cultural distance. A study of 20 European countries found that the degree to which a person values cultural homogeneity is a much more accurate predictor of their attitudes towards immigrants than their economic standing (Sides and Citrin 2007; Burns and Gimpel 2000).

Political orientation has also been found to be significant in determining people's ATII. In general, people who identify with the political right are more likely to support restrictive immigration policies (Hainmueller and Hiscox 2007; McLaren 2001; McLaren 2003; Semyonov et al. 2006; Semyonov et al. 2008) and favor excluding immigrants from equal access to institutions as native-born citizens, such as healthcare or welfare (Gorodzeisky and Semyonov 2009). Furthermore, in some studies, the effect of political orientation remains even after controlling for other factors (Kunovich 2009; Semyonov 
et al. 2006; Knoll et al. 2011). Hainmueller and Hangartner exploit the natural experiments of Swiss local elections which, prior to 2003, could include referendums to determine which applicants should be granted Swiss citizenship (Hainmueller and Hangartner 2013).

Finally, religion has been found in various studies to be significant in determining attitudes but the research is inconclusive, as religion has been found to be have both positive (Scheepers et al. 2002) and negative impact on anti-immigrant sentiment (Mayda 2006). Studies often differ in how they define and measure religion, with some studies looking at which specific sect people ascribe to, e.g. Catholic, Protestant, Muslim, and others measuring church attendance or a subjective measure of how important religion is to the respondent (Scheepers et al. 2002). Thus, results are not always comparable. In a crossnational study, Scheepers et al. find that religion exerts a similar effect across countries, but how the individual expresses or understands their religion greatly influences the result (Scheepers et al. 2002). Those who subscribed to a formal religion were found to have greater prejudice than those who were non-religious. Christians, both Catholics and Protestants, showed the most anti-immigrant sentiment. Moreover, those who expressed a greater level of religious particularism or valued doctrinal adherence were also more likely to express prejudice against ethnic minorities. However, respondents who answered that religiosity was more important in their lives and who felt more strongly that they had a spiritual life (as compared to an intellectual or emotion life) were found to have lower levels of prejudice (Scheepers et al. 2002).

\section{Group-level}

Group-level factors are factors which relate to an individual's attachment to a group and where the primary concern is the group's interests rather than the individual's selfinterest. Studies which advance these theories generally rely on two theoretical foundations: the concept of intergroup conflict and the concept of social identity theory. Intergroup conflict theory states that prejudice between groups arises due to real or perceived conflicts over a limited amount of resources, either material resources or more symbolic resources, like esteem and social value (Esses et al. 1998; Smith et al. 2015). In contrast to intergroup conflict theory, social identity theory centers the primacy of identities in shaping opinions towards others and other groups. It states that individuals categorize other people into either an outgroup (for those who are determined to be different from themselves) and an ingroup (for themselves and those who are similar to them). Limiting the opportunities of those in the outgroup becomes one strategy to increase the distinctiveness and cohesion of the ingroup (Esses et al. 1998). Social identity theory posits that people who more strongly identify with the nation and who perceive threat more strongly will have a more negative reaction towards immigrants. National identity, meaning the degree to which a person is attached to their country, has been found to have a positive relationship with more exclusionary attitudes towards immigrants and immigration (Kunovich 2009; Coenders and Scheepers 2004; Mummendey et al. 2001; Gijsberts and Hagendoorn 2017).

\section{Data}

This study uses data from the sixth wave of the World Values Survey, conducted between 2010 to 2014 (Inglehart et al. 2014). The World Value Survey seeks to measure 
and track "the beliefs, values and motivations of people throughout the world" (WVS Database 2020) and asks respondents over 200 questions related to topics such as religion, governance, social relations, morality, and so on. The survey employs a common questionnaire that is translated by a national team composed of social science researchers from universities and/or research institutes. The national team then conducts a nationally representative survey of residents 18 and older, regardless of their nationality, to reach a minimum sample size of at least 1200 people (Inglehart et al. 2014). The data from the following nine countries and territories, four in Southeast Asia and five in East Asia, was used in this study: China, Hong Kong, Japan, Malaysia, the Philippines, Singapore, South Korea, Taiwan, and Thailand.

Immigrants were eliminated as the study aims to understand the attitudes and reactions of autochthonous populations towards immigrants. China, Hong Kong, and Japan did not record if any respondent was an immigrant. In the case of China and Japan, the inclusion of immigrants is unlikely to be big, as immigrants accounted for only $0.06 \%$ and $1.91 \%$ of the population respectively (UN DESA 2013). While the inclusion of these respondents is not ideal and creates some noise, it is unlikely to greatly alter the results. Hong Kong is a more complicated case, as the immigrant population is much larger and because the question of who is an immigrant is deeply political. According to the UN DESA data, $83.8 \%$ of the immigrant population in Hong Kong comes from mainland China or Macao; immigrants who are not from a Chinese territory make up 6.3\% of the total Hong Kong population (UN DESA 2013). However, because the question of who is an immigrant and who is part of the autochthonous population is so political, it is likely that the definition of who is and is not an immigrant varies more widely amongst respondents in Hong Kong than in other countries in the sample. Therefore, some of the results from Hong Kong may be more open to bias and noise than that from other countries.

Finally, respondents who did not respond to the dependent variable were also eliminated from the sample. In total, the combined number of respondents for this study was 12,119 .

The dependent variable asks respondents about prioritizing autochthonous people in the hiring process. The question was recoded so that a response [1] would indicate a more exclusionary attitude towards immigrants and [0] would indicate a more lenient or inclusive attitude. The survey questions is as follows:

V46. When jobs are scarce, employers should give priority to people of this country over immigrants.

\section{[1] Agree \\ [0] Neither "originally coded [2] \\ [0] Disagree *originally coded [3]}

Figure 1 shows the distribution of respondents for each country who responded negatively (shown in green) towards immigrants in the dependent variable. For some countries, a majority of respondents responded negatively towards the question, raising the possibility of class imbalance in the multiple logistic regression during the pre-selection. Peduzzi et al. (1996) and Steyerberg et al. (1999) recommend that a sample include at least 10 events per variable (EPV) to account for class imbalance. Using this 


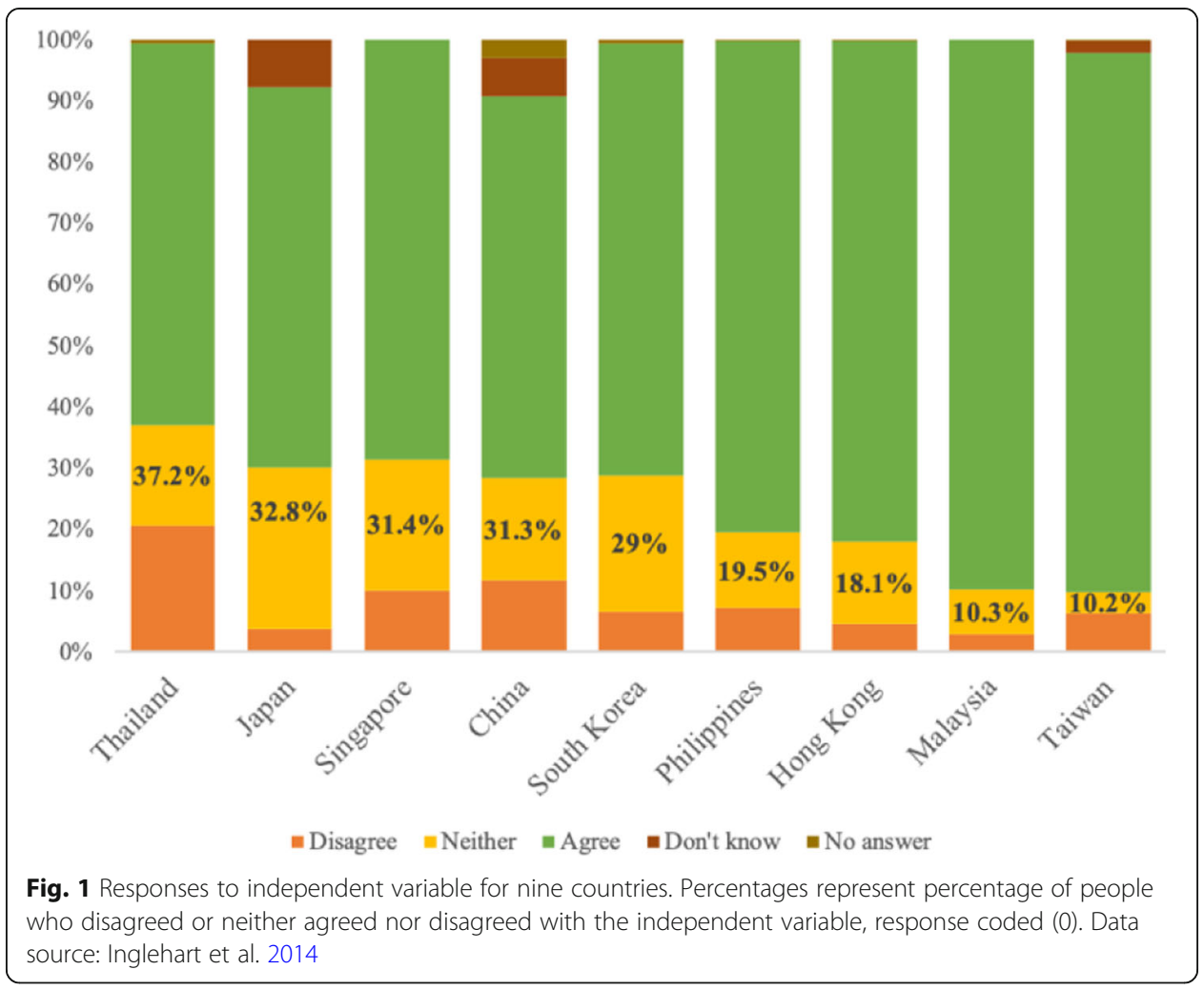

heuristic, Taiwan, Hong Kong, and Singapore may be vulnerable to bias and overestimated regression coefficients during multiple logistic regression. However, when obtaining the edge weights in the network analysis section, a different subset of the data is used, and the samples are not susceptible to this bias.

\section{Methodology}

Table 3 shows a summary of the methodology used in this study. The methodology is divided into two distinct stages: pre-selection and network analysis. The pre-selection applies a series of statistical tests in order to determine which attitudinal variables are

Table 3 Stages, methods and their description of the study

\begin{tabular}{|c|c|c|}
\hline Stage & Methodology & Description \\
\hline \multirow[t]{4}{*}{ Pre-selection } & 1. Spearman's correlation & \\
\hline & 2. Chi-squared test of independence & \\
\hline & 3. Linear regression of continuous variables & \\
\hline & 4. Multiple logistic regression & \\
\hline \multirow[t]{7}{*}{ Network Analysis } & 1. Link weight in bipartite network & i. Logistic regression \\
\hline & 2. 1. 1. One-mode projection: country network & i. Normalization \\
\hline & & ii. Matrix multiplication \\
\hline & & iii. Community detection: Louvain method \\
\hline & 3. 1. 1. One-mode projection: variable network & i. Normalization \\
\hline & & ii. Matrix multiplication \\
\hline & & iii. Community detection: Blockmodeling \\
\hline
\end{tabular}


the most significant in determining attitudes towards immigrants in the nine countries. Attitudinal variables, education, and demographic variables are then regressed against the response variable to identify significant variables.

Once a set of significant variables are identified, a bipartite network is created to represent the relationship between the selected variables and the dependent variable for each country. This bipartite network is then projected, creating two one-mode networks: a country network and a variable network. The country network allows for comparison of how the countries are similar or dissimilar in the determinants of attitudes towards immigrants. The variable network shows how variables reinforce or negate one another in determining attitudes. Blockmodeling was applied to the variable network find the determinants, or clusters of correlated variables, of attitudes towards immigrants.

\section{Pre-selection method}

Given the wide range of attitudinal questions included in the World Values Survey, it is necessary to eliminate variables which are not significantly related to the dependent variable before conducting network analysis. Previous studies generally pre-select variables based on the theoretical or empirical literature; however, in order to allow for the inclusion of variables which may have been overlooked by previous studies or which are less relevant in European and Settler countries, this study pre-selects variables by conducting a series of statistical tests which seek to identify attitudinal variables which have a significant relationship with the dependent variable.

Variables were divided into three categories: demographic variables, the education variable, and attitudinal variables. Demographic variables included variables for subjective social class, subjective income, sex, age, town size, and political orientation. The education variable measured the highest variable attained. These variables were automatically included in the multiple logistic regression, due to their support in the literature. Attitudinal variables were subjected to a series of statistical tests. The final step of the pre-selection process was a multiple logistic regression including demographic, education, and attitudinal values, with variables deleted according to backwards deletion. Significant variables were then included in the network analysis. The pre-selection method was repeated for each country individually, to find the variables most relevant to attitudes towards immigrants in each country. The following section describes the pre-selection of attitudinal variables will be discussed in more depth.

First, the pre-selection sought to eliminate variables that were highly correlated with one another using Spearman's rank correlation. Variables were considered highly correlated if they had a rank correlation coefficient greater than absolute value of 0.75 . This value was chosen to avoid including redundant variables in the network analysis. Increasing the cut-off above 0.75 had little to negligible effect on the variables selected. Variables, $X=\left\{X_{i}\right\}(i=1, \cdots, N)$ and $Y=\left\{Y_{i}\right\}(i=1, \cdots, N)$, were converted to rank variables $r_{X}$ and $r_{Y}$. $N$ is the number of the responses. Variables, $X$ and $Y$, were considered highly correlated if

$$
0.75 \leq\left|\operatorname{cor}\left(r_{X}, r_{Y}\right)\right|=\left|\frac{\operatorname{cov}\left(r_{X}, r_{Y}\right)}{\sigma_{r_{X}} \sigma_{r_{Y}}}\right|
$$

where cor is the correlation of rank variables $r_{X}$ and $r_{Y}$, or is equal to the covariance of the rank variables divided by the standard deviation $\sigma_{r_{X}}$ and $\sigma_{r_{Y}}$. If a pair of variables were found to be highly correlated, then logistic regression against the dependent 
variable was used to determine which variable, $X$ or $Y$, had a more statistically significant relationship with the response variable measuring attitudes towards immigrants, $P$.

$$
\log \left(\frac{P_{i}}{1-P_{i}}\right)=\alpha^{(0)}+\alpha^{(1)} X_{i}+\varepsilon_{i}
$$

where $P=\left\{P_{i}\right\}(i=1, \cdots, N)$ is the probability that the dependent variable $Y$ will equal 1. Statistical significance with the dependent variable was judged by the Akaike Information Criterion (AIC) of the logistic regression model, as defined by

$$
A I C=-2 \ln (L)+2 s
$$

where $L$ is the value of the likelihood and $k$ is the number of estimated parameters. The variable for which the model's AIC was smaller was retained, while the other variable in the pair was eliminated. As both variables have an equal number of parameters $(s=1)$, the variable with the larger $L$, was selected.

In the second step of the pre-selection, a chi-squared test of independence was used to eliminate insignificant variables. The chi-squared test was calculated according to the formula

$$
\chi^{2}=\sum_{i=1}^{M} \frac{\left(O_{i}-E_{i}\right)^{2}}{E_{i}}
$$

where $O=\left\{O_{i}\right\}(i=1, \cdots, M)$ is the observed frequency of responses to the dependent variable for each response, and $E=\{E\}(i=1, \cdots, M)$ is the expected frequency of responses to the dependent variable for each response. Here $M$ is the number of possible responses. Under the assumption of no association, the null hypothesis, the probability of negative attitudes towards immigrants would be similar regardless of the response to the independent variable. Variables for which the $p$-value of $x^{2}$ was less than or equal to 0.05 , given the degrees of freedom, were retained, while variables where the null hypothesis could not be rejected were eliminated.

Continuous variables - defined as variables with more than three possible selection of the responses - were subjected to an additional test. The aim of this test was to eliminate variables for which there was not a linear relationship with the dependent variable. In this case, the frequency of exclusionary attitudes towards immigrants for each response to the dependent variable was regressed against the responses to the dependent variable, $Y$. $Y_{i}$ was the proportion of exclusionary attitudes for each response $i$, to the dependent variable. For each

$$
Y_{i}=\beta^{(0)}+\beta^{(1)} X_{i}+\varepsilon_{i}
$$

where $X_{i}$, and $\beta$ are the parameters of the model. If the $p$-value of the model was greater than 0.05 , the dependent variable was eliminated.

In the final step of the pre-selection, demographic, education, and attitudinal variables were regressed against the dependent variable in a multiple logistic regression.

$$
\log \left(\frac{P_{i}}{1-P_{i}}\right)=\gamma^{(0)}+\sum_{j=1}^{V^{\prime}} \gamma^{(j)} X_{i}^{(j)}+\varepsilon_{i}
$$

where $P=\left\{P_{i}\right\}(i=1, \cdots, N)$ is the probability that the dependent variable $Y$ will equal 1 , given the dependent variable $X$, and $\gamma$ are the parameters of the model. Variables 
were eliminated via stepwise backwards deletion. In backwards deletion, models begin with all candidate variables. The variable with the highest $p$-value is eliminated, and the model is run again. This process was repeated until all variables showed significance of or below $5 \%$. When all variables in the multiple logistic regression were significant above 0.05 , these variables were selected for the network analysis.

Following this pre-selection method, a set of sets resulted in a total of 45 relevant variables for network analysis.

\section{Network analysis}

Having selected the variables, a bipartite network was then constructed, with a class of nodes for countries and a class of nodes for variables. The weight of the edges between the classes is determined by the regression coefficient of the independent variables on the dependent variable for each country. In short, the bipartite network shows the effect of each variable on the response variable for each country. The weights of the edges between countries and variables were obtained by logistic regression.

First, variables were rescaled according to min-max normalization, as independent variables had different ranges of responses, from binary questions to questions with up to ten possible responses. 18 of the 45 variables were binary questions, and the remaining 27 were multi-level questions. This step may cause coefficients multi-level questions to be underestimated, but allows for comparison between variables.

$$
X_{i}^{\prime}=\frac{X_{i}-\min (X)}{\max (X)-\min (X)}
$$

For a variable $X$, each element $X_{i}$ was linearly transformed to $X_{i}^{\prime}$, so that responses ranged from 0 to 1 .

The edge weights, $\delta_{c}^{(j)}$ between country node c and variable node $\mathrm{j}$ were obtained by the following formula:

$$
\log \left(\frac{P_{i}}{1-P_{i}}\right)=\delta^{(0)}+\delta_{c}^{(j)} X_{i}^{(j)}+\varepsilon_{i}
$$

where $P=\left\{P_{i}\right\}(i=1, \cdots, N)$ is the probability that the dependent variable $Y$ will equal 1 , and $\delta$ are the parameters of the model. If the $p$-value of the model is greater than 0.05 , then the edge weight is equal to 0 .

Using the parameters of the simple logistic regression, a bipartite weighted adjacency matrix was created, $\mathrm{A}=\left\{a_{i j}\right\}=\left\{\delta_{i}^{(j)}\right\} \quad(i=1, \cdots, C ; j=1, \cdots, V)$. Here $V$ is the number of selected variables. The edge weights $a_{i j}$ were created by the parameters of the simple logistic regression. This weighted adjacency matrix is provided in Appendix G.

In order to understand the relationships between variables within the same class, i.e. the relationships between countries (variables), it is necessary to make two onemode projections of the network, first of the country class and then of the variable class. These one-mode projections multiply country (variable) vectors by other country (variable) vectors to produce a scalar value representing the similarity between the two vectors. 
First, the matrix was rescaled so that after matrix multiplication, the dot product between two countries or two variables would be on a scale from -1 to 1 . Matrix A is rewritten using row-wise country vector $c^{(i)}(i=1, \cdots, C)$ :

$$
\mathrm{A}=\left[\begin{array}{c}
c^{(1)} \\
c^{(2)} \\
\vdots \\
c^{(C)}
\end{array}\right]=\left\{c_{j}^{(i)}\right\}
$$

Each country vector $c^{(i)}$ was normalized according to the following formula:

$$
\hat{c}^{(i)}=\frac{c^{(i)}}{\sqrt{\sum_{j=1}^{V} c^{(i)}{ }_{j}^{2}}}
$$

Having normalized the country vectors, the normalized matrix

$$
\hat{\mathrm{A}}=\left[\begin{array}{c}
\hat{c}^{(1)} \\
\hat{c}^{(2)} \\
\vdots \\
\hat{c}^{(C)}
\end{array}\right]=\left\{\hat{c}^{(i)}{ }_{j}\right\}
$$

was multiplied by its transposed normalized matrix $\hat{\mathrm{A}}^{T}$, in order to find the degree of similarity between countries $\Phi=\left\{\phi_{i j}\right\}(i=1, \cdots, C ; j=1, \cdots, C)$.

$$
\begin{aligned}
& \Phi=\hat{\mathrm{A}} \cdot \hat{\mathrm{A}}^{T} \\
& \phi_{i j}=\sum_{k=1}^{V} \hat{c}^{(i)}{ }_{k} \hat{c}^{(j)} k
\end{aligned}
$$

The result of this operation is a weighted adjacency matrix of the one-mode projection of countries $\left\{\phi_{i j}\right\}$, shown in Appendix F.

The same process was then repeated to find which predictor variables showed greater similarity. Matrix A is rewritten using column-wise variable vector $v^{(j)}(j=1, \cdots, V)$ :

$$
\mathrm{A}=\left[\begin{array}{llll}
v^{(1)} & v^{(2)} & \cdots & v^{(V)}
\end{array}\right]=\left\{v_{i}^{(j)}\right\}
$$

Each variable vector $v^{(j)}$ was normalized according to the following formula:

$$
\hat{v}^{(j)}=\frac{v^{(j)}}{\sqrt{\sum_{j=1}^{C} v_{i}^{(j)^{2}}}}
$$

Having normalized the variable vectors, the normalized matrix

$$
\hat{\mathrm{B}}=\left[\begin{array}{llll}
\hat{v}^{(1)} & \hat{v}^{(2)} & \cdots & \hat{v}^{(V)}
\end{array}\right]=\left\{\hat{v}_{i}^{(j)}\right\}
$$

multiplies its transposed normalized matrix $\hat{\mathrm{B}}^{T}$, in order to find the degree of similarity between countries $\Psi=\left\{\psi_{i j}\right\}(i=1, \cdots, V ; j=1, \cdots, V)$.

$$
\Psi=\hat{\mathrm{B}}^{T} \cdot \hat{\mathrm{B}}
$$




$$
\psi_{i j}=\sum_{k=1}^{C} \hat{v}_{k}^{(i)} \hat{v}_{k}^{(j)}
$$

This produced the weighted adjacency matrix of the one-mode projection of variables $\left\{\psi_{i j}\right\}$, shown in Appendix G.

In order to detect communities within the network, a Louvain clustering was applied (Blondel et al. 2008). The Louvain method optimizes modularity, which is defined as

$$
\begin{aligned}
& Q^{(C)}=\frac{1}{2 m^{(C)}} \sum\left[\phi_{i j}-\frac{k^{(C)}{ }_{i} k^{(C)} j}{2 m^{(C)}}\right] \delta\left(c_{i} c_{j}\right) \\
& Q^{(V)}=\frac{1}{2 m^{(V)}} \sum\left[\psi_{i j}-\frac{k^{(V)}{ }_{i} k^{(V)} j}{2 m^{(V)}}\right] \delta\left(c_{i} c_{j}\right)
\end{aligned}
$$

Here $\phi_{i j}$ represents the edge weight between nodes $i$ and $j, k^{(C)}{ }_{i}$ is the sum of weights attached to node $i, k^{(C)}$ is the sum of weights attached to node $j, m^{(C)}$ is the sum of edge weights in the country network. $\psi_{i j}$ represents the edge weight between nodes $i$ and $j, k^{(V)}{ }_{i}$ is the sum of weights attached to node $i, k^{(V)}{ }_{j}$ is the sum of weights attached to node $j, m^{(V)}$ is the sum of edge weights in the variable network. The Kronecker's delta is defined as follows

$$
\delta=\left\{\begin{array}{cc}
1 & \left(c_{i}=c_{j}\right) \\
0 & \left(c_{i} \neq c_{j}\right)
\end{array}\right.
$$

where $c_{i}$ is the community of node $i$ and $c_{j}$ is the community of node $j$.

Because of the presence of negative ties between variable nodes in the one-mode projection, Louvain clustering could not be used. For this reason, blockmodeling was employed instead. Blockmodeling is a technique which permutes the order of nodes in an adjacency matrix to find clusters. As the network is signed, Doreian and Mrvar's relaxed structural balance blockmodel, a generalization of structural balance blockmodels, was used (2009). The relaxed structural balance blockmodel also seeks to optimize a criterion function. In the case of this study, the following criterion function was used:

$$
P\left(C_{k}\right)=\rho \mathcal{N}+(1-\rho) \mathcal{P}
$$

$P\left(C_{k}\right)$ equals the sum of inconsistencies which violate structural balance in the network, given $k$ number of clusters, $C$. $\mathcal{P}$ represents the total number of positive ties in a negative block, $\mathcal{N}$ the total number of negative ties in positive blocks, and $0 \leq \rho \leq 1$. This criterion function resembles Harary et al.'s (1965) line index of imbalance; however, $\rho$ allows for positive ties in negative blocks and negative ties in positive blocks to be weighted differently. When $\rho=0.5$, negative and positive inconsistencies are equally weighted. In the case of this study, $\rho=0.75$, as the aim was to prioritize consistency within blocks over consistency between blocks.

As the criterion function decreases monotonically (Doreian and Mrvar 2009), it is necessary to dictate the number of clusters. In order to determine the number of clusters, the eigenvectors of the correlation matrix of the variables were taken. Using the eigenvalues, it was possible to determine the number of eigenvectors, $n$, were necessary to explain a majority of the variance in the model. Given that for each eigenvector a 
variable could have either a positive or negative sign, $k$ number of clusters would be sufficient to explain a majority of the variance in the model.

$$
k=2^{n}
$$

Finally, to determine the representative variable of each block, the node strength of the variable within the block was taken. The node strength is the sum of the weights of ties from a variable to other variables within its cluster. Through this method, the most central variable within the cluster was found. A keyword was then chosen by examining the survey question of the most central variable and the other variables in the block and referring to the classifications provided by the World Values Survey. In several blocks, the node with the highest node strength was part of a series of survey questions which also appear in the block. These series are often used to measure a general tendency, like prejudice or group identification. In this case, the keyword was taken from the series of questions.

\section{Results}

Table 4 lists how many variables were eliminated in each step of the pre-selection process for each country.

The variables removed for each country in the Spearman's correlation are provided in Appendix A, showing both the rho correlation between the variables and the respective AIC of a logistic regression between the variable and the response variable. Because of the high correlation between the variables, the AIC from each regression are very similar, with some notable exceptions. An average of 8 variables were removed during this step. The chi-squared test between variables and the response variable eliminated far more variables, with an average of 80 variables removed for each country in this step. The results of the chi-squared tests are listed in Appendix B. Simple linear regression of the proportion of negative attitudes against continuous variables also eliminated a large number of variables. R-squared values are unusually high, due to the low number of data points. The results of these regressions are listed in Appendix $\mathrm{C}$, showing the $\mathrm{r}$-squared, coefficient, standard error, $\mathrm{t}$-value, and $p$-value.

The final multiple logistic regression results are provided in Appendix D. Measure of overall fit of the model, such as AIC and pseudo $\mathrm{R}^{2}$, are provided as well as parameter estimations. Overall fit of the models can be considered strong when compared with similar regression models of attitudinal determinants of attitudes. The average pseudo $R^{2}$ is 0.169 , ranging from a minimum of 0.115 to a maximum of 0.342 . Regression models using survey data to measure attitudes often perform somewhat poorly on some measures of performance (see r-squared values in Mayda 2006; O'Rourke and Sinnott 2006), as a variety of factors determine attitudes, including factors like social influences, media influence, mood, etc., which cannot be measured by the survey. As individual variable are unlikely to explain a large amount of the observed variance, $p$-value was used to identify variables correlated with the dependent variable.

Having selected the variables, a weighted adjacency matrix was created for the bipartite network of countries and variables, as shown in Appendix E. Simple logistic regression regressed against the response variable provided the edge weights, with variables that did not show statistical significance given an edge weight of 0 . This matrix was then normalized and multiplied using the dot product to create a one-mode projection of countries and variables. These matrices can be found in Appendix F and Appendix $\mathrm{G}$, respectively. 


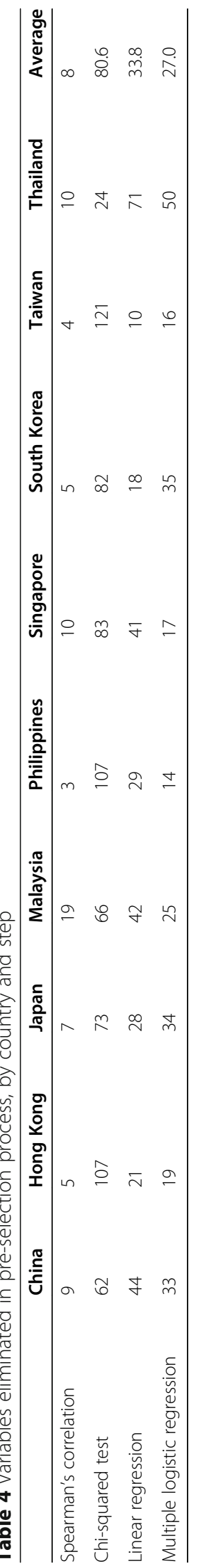




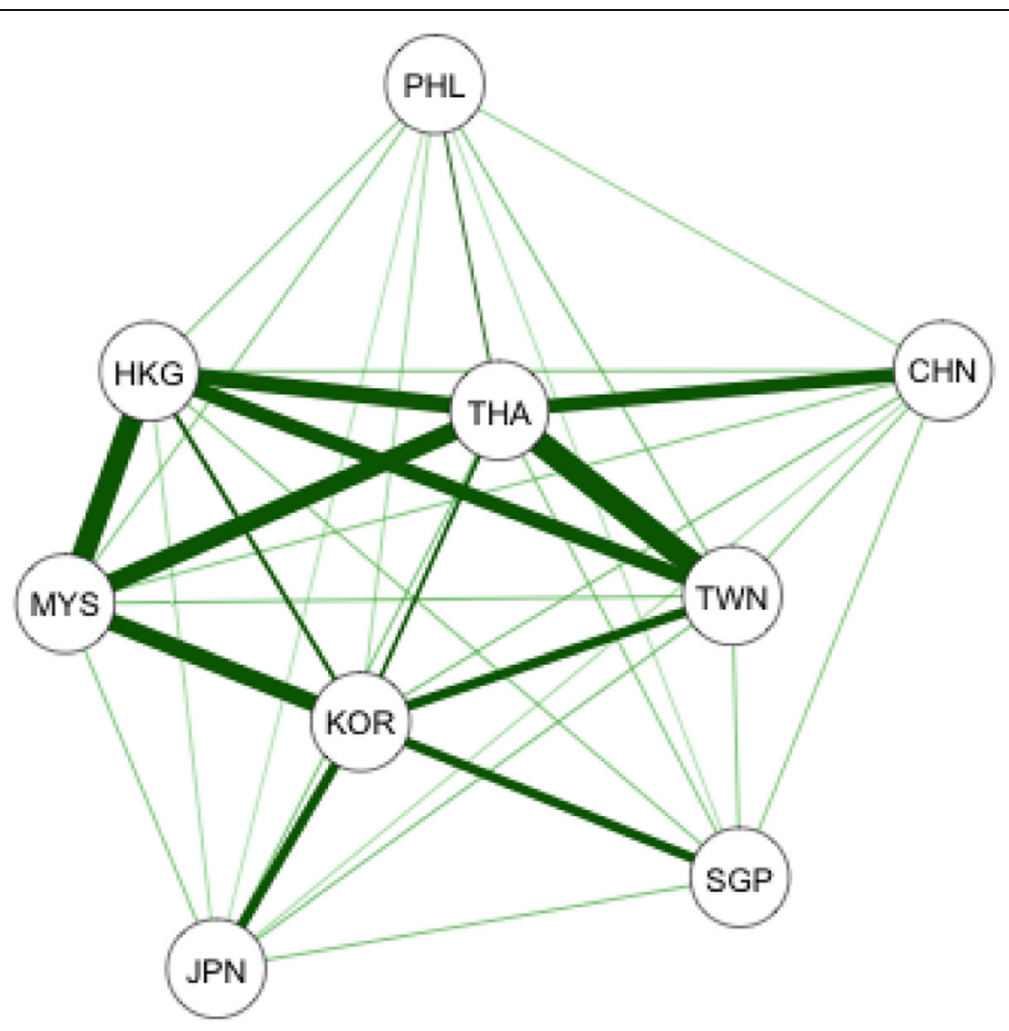

Fig. 2 Country network. Green links represent positive ties. Edge weight represented by width of edge

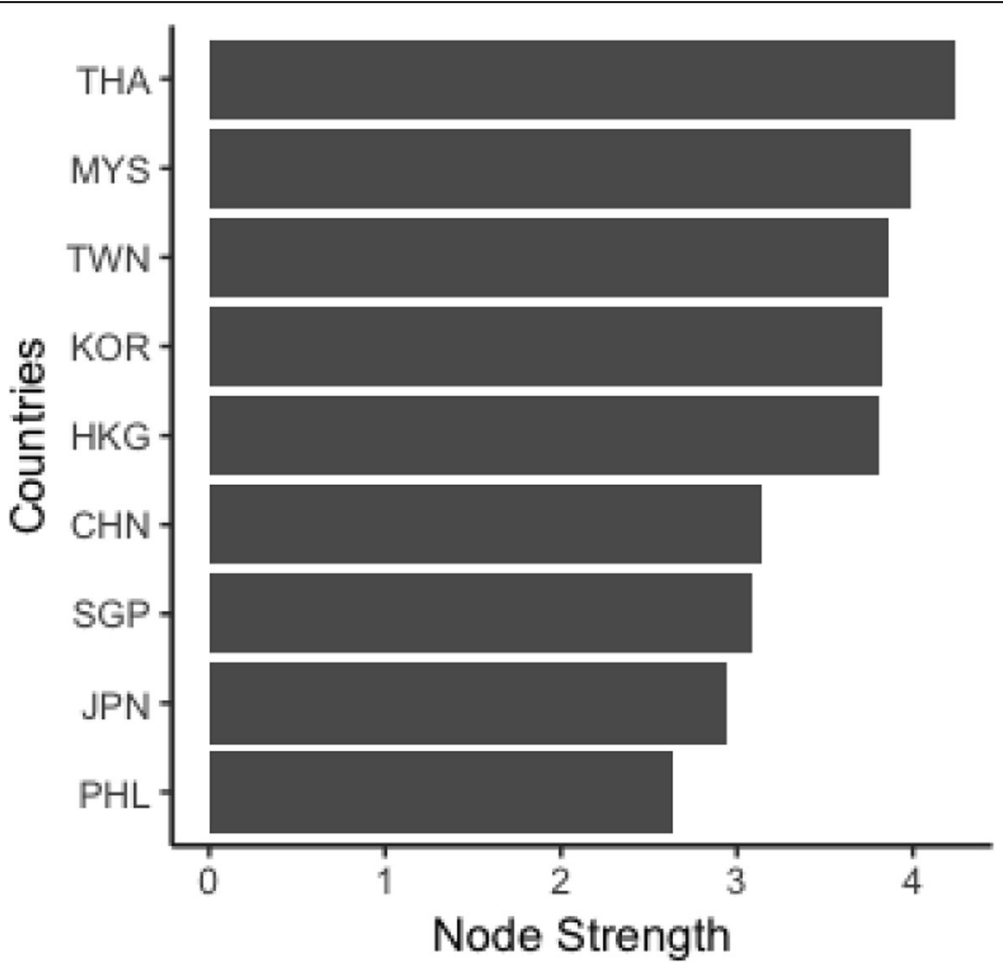

Fig. 3 Node strength of country network 


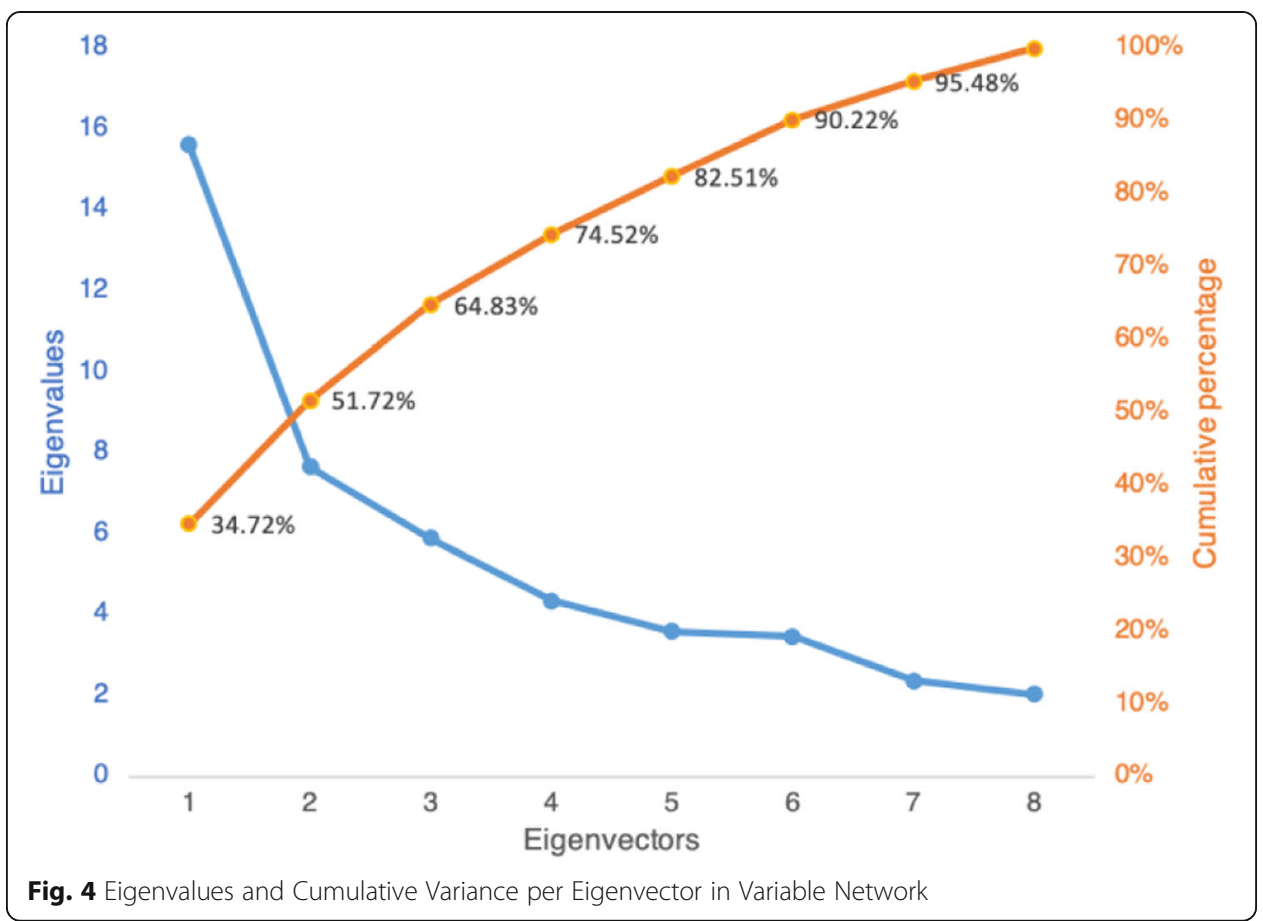

\section{Countries}

Figure 2 shows the network of countries that is the result of the one-mode projection of the bipartite graph. Green edges represent positive ties between countries, and the weight of the edges are represented by the width of the edge. The graph is a clique, meaning that all countries have ties with all other countries. All edges are positive, meaning that countries are similar in their determinants of attitudes. A Louvain clustering algorithm was applied to detect any communities within the network. Dividing the network in two clusters resulted in a negative, though negligible, modularity score $(-2.05 \mathrm{e}-16)$, meaning that keeping the network as a single cluster is the optimal partition of the network.

Some of the edges between countries have larger weights, or show a greater degree of similarity between countries. Summing the weights of ties from a country (node strength) can help illustrate which countries show a large degree of similarity to the rest of the countries in the sample and which countries are fairly unique.

As shown by Fig. 3, countries Thailand, Malaysia, Taiwan, and Korea have the largest node strength. The Philippines had the lowest node strength, meaning it is the most unique country with regards to its determinants of attitudes

\section{Variables}

Figure 4 shows the cumulative percentage of variance for each eigenvector of the correlation matrix of the variable network. Given the cumulative percentage of variance, three eigenvectors are sufficient to explain $64.83 \%$ of the variance in the model. As each variable could be either negatively or positively signed in each vector, this suggests that eight clusters $\left(2^{3}\right)$, would be sufficient to explain $64.83 \%$ of the variance of the model. 


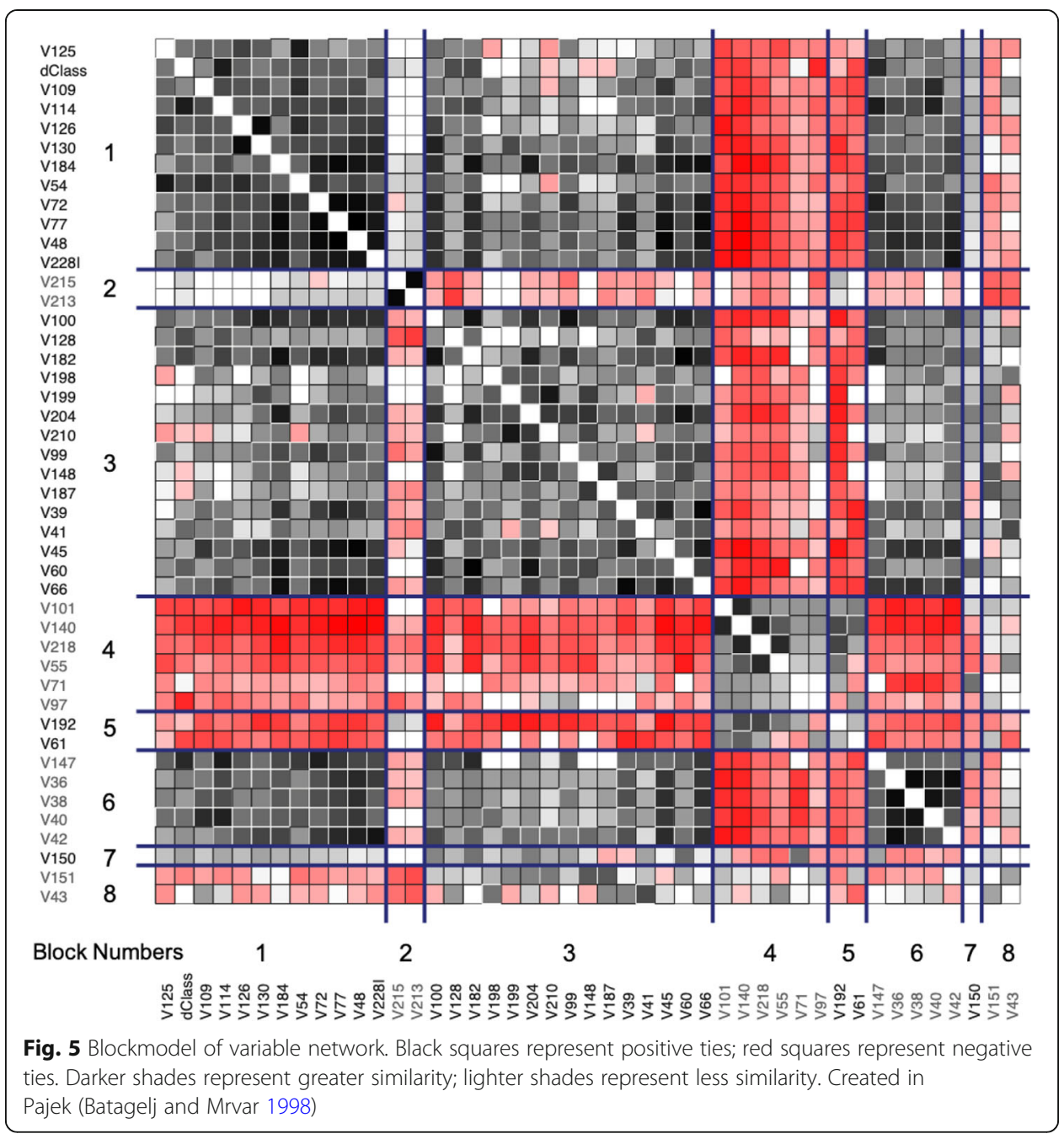

Having specified that eight clusters would be sufficient, the following blockmodel (Fig. 5) was created.

The blockmodel created eight blocks of variables, henceforth called determinants. Between variables clustered in the same blocks, only two negative ties were found. Both of these negative ties were found in Block 3, one between V41 and V210 and the other between V41 and V199. The total inconsistency score for the partition, or $P(C)$, was 4.930.

When the variables in the variable network are clustered by block, the network shown in Fig. 6 is created.

Finally, the blockmodel shown in Fig. 5 can be reduced to an image matrix, showing the overall signs of the blocks. This image matrix is shown in Table 5. "P" represents positive blocks, in which the majority of blocks are positive, and "N" represents negative blocks. Positive blocks off the diagonal are considered an error when structural balance is not relaxed and are filled with grey.

Having described the overall structure, each block was then examined in more detail. The following tables provide the survey question to which each variable refers, ordered from greatest to smallest node strength. The keyword to define each determinant is 


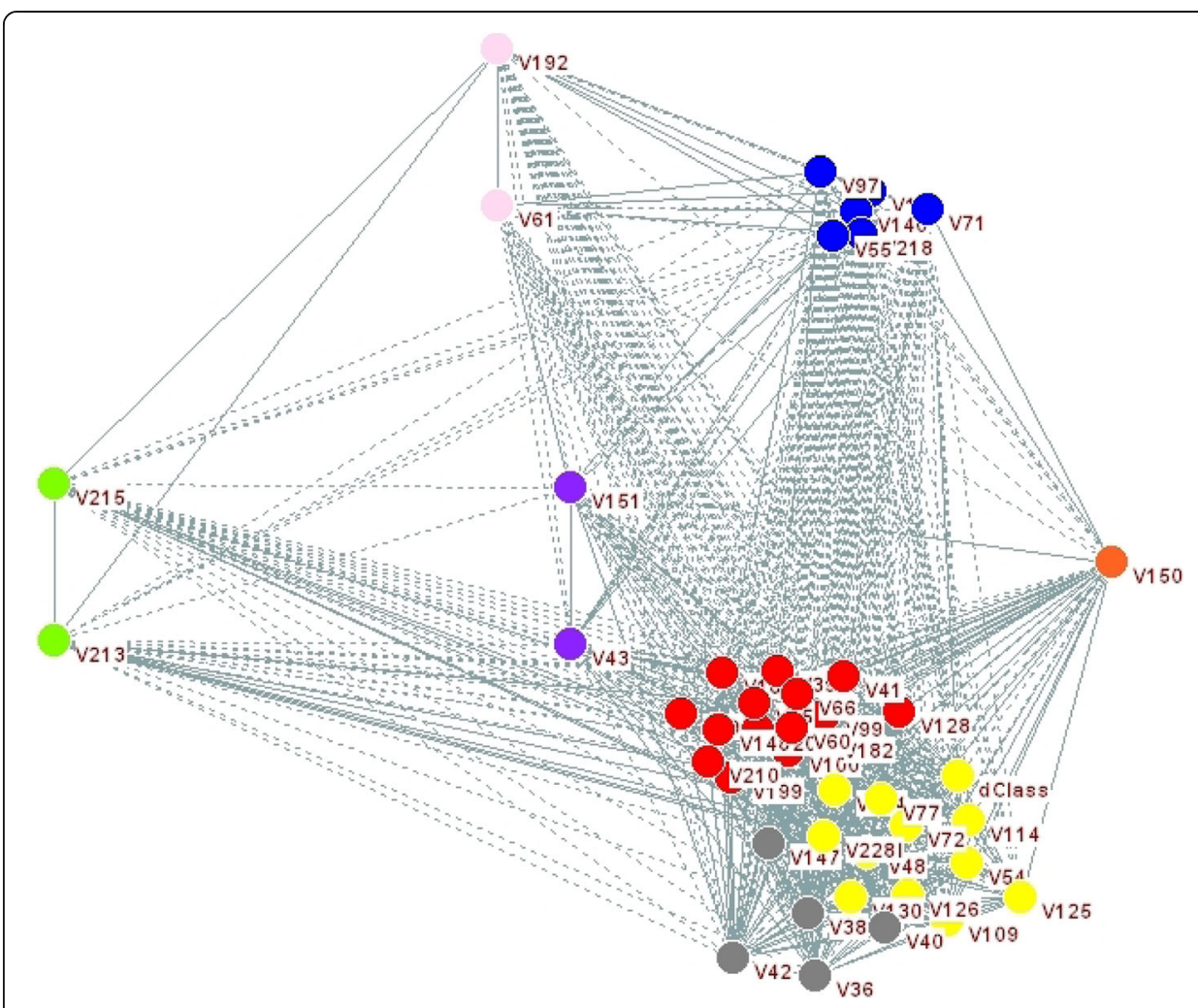

Fig. 6 Variable network with clusters from blockmodel. Colors represent clusters dictated by blockmodel. Positive ties represented by solid lines; negative ties represented by dashed lines

also described. The scalar value of similarity between variables, as obtained by the onemode projection, is sometimes discussed.

Block 1 represents variables related to independence and social dependencies. The cluster also includes questions related to normative behavior (V77), confidence and trust in various institutions (V228I, V126, V114, V109, V125), and a variable related to

Table 5 Image matrix of blockmodel

\begin{tabular}{l|llllllll} 
& 1 & 2 & 3 & 4 & 5 & 6 & 7 & 8 \\
\hline 1 & $\mathrm{P}$ & $\mathrm{P}$ & $\mathrm{P}$ & $\mathrm{N}$ & $\mathrm{N}$ & $\mathrm{P}$ & $\mathrm{P}$ & $\mathrm{N}$ \\
2 & $\mathrm{P}$ & $\mathrm{P}$ & $\mathrm{N}$ & $\mathrm{N}$ & $\mathrm{P}$ & $\mathrm{N}$ & $\mathrm{P}$ & $\mathrm{N}$ \\
3 & $\mathrm{P}$ & $\mathrm{N}$ & $\mathrm{P}$ & $\mathrm{N}$ & $\mathrm{N}$ & $\mathrm{P}$ & $\mathrm{P}$ & $\mathrm{P}$ \\
4 & $\mathrm{~N}$ & $\mathrm{~N}$ & $\mathrm{~N}$ & $\mathrm{P}$ & $\mathrm{P}$ & $\mathrm{N}$ & $\mathrm{N}$ & $\mathrm{P}$ \\
5 & $\mathrm{~N}$ & $\mathrm{P}$ & $\mathrm{N}$ & $\mathrm{P}$ & $\mathrm{P}$ & $\mathrm{N}$ & $\mathrm{N}$ & $\mathrm{N}$ \\
6 & $\mathrm{P}$ & $\mathrm{N}$ & $\mathrm{P}$ & $\mathrm{N}$ & $\mathrm{N}$ & $\mathrm{P}$ & $\mathrm{N}$ & $\mathrm{N}$ \\
7 & $\mathrm{P}$ & $\mathrm{P}$ & $\mathrm{P}$ & $\mathrm{N}$ & $\mathrm{N}$ & $\mathrm{N}$ & $\mathrm{P}$ & $\mathrm{P}$ \\
8 & $\mathrm{~N}$ & $\mathrm{~N}$ & $\mathrm{P}$ & $\mathrm{P}$ & $\mathrm{N}$ & $\mathrm{N}$ & $\mathrm{P}$ & $\mathrm{P}$ \\
\hline
\end{tabular}


Table 6 Survey questions: Block 1 - Independence

\begin{tabular}{lll}
\hline Variable & Survey Question & $\begin{array}{l}\text { Node } \\
\text { Strength }\end{array}$ \\
\hline V48 & Having a job is the best way for a woman to be an independent person & 8.224 \\
V72 & $\begin{array}{l}\text { Living in secure surroundings is important to this person; to avoid anything that might } \\
\text { be dangerous. }\end{array}$ & 7.897 \\
V77 & $\begin{array}{l}\text { It is important to this person to always behave properly; to avoid doing anything } \\
\text { people would say is wrong. }\end{array}$ & 7.881 \\
V54 & [Do you agree that] being a housewife is just as fulfilling as working for pay? & 7.719 \\
V228I & Voters are offered a genuine choice in elections & 7.694 \\
V126 & [H]ow much confidence [do] you have in [the United Nations] & 7.446 \\
V130 & [ls having a democratic political system] a very good way of governing a country & 7.443 \\
V114 & [H]ow much confidence [do] you have in [the courts] & 7.441 \\
V184 & To what degree are you worried about [a terrorist attack] & 7.213 \\
dClass & Would you describe yourself as belonging to the [upper class, upper middle class, & 6.543 \\
& lower middle class, working class, lower class] & \\
V109 & [H]ow much confidence do you have in [the armed forces] & 6.464 \\
V125 & [H]ow much confidence you have in [regional organizations] & 5.902 \\
\hline
\end{tabular}

class. In addition, V72 and V184 relate to security and anxiety amongst respondents. The question which each variable represents is detailed in Table 6 .

As can be seen from Fig. 7, Block 1 is a clique, meaning that all the nodes are connected by edges to all other nodes. All ties are positive, showing that the variables are correlated with other variables in the block.

Block 2 contains two variables from a series regarding identification with a group, as seen in Table 7. Respondents were asked whether they see themselves as part of their local community (V213) and part of larger, political organizations, namely their regional organizations (V215). As Block 2 only contains two variables, the node strength

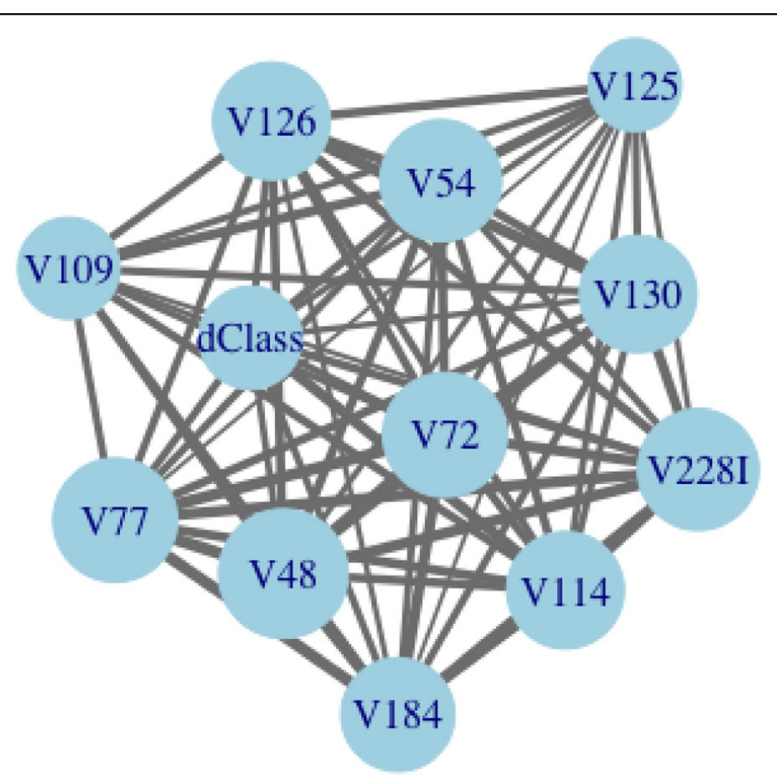

Fig. 7 Network of variables in Block 1. Positive ties represented by grey links; negative ties by red ties 
Table 7 Survey questions: Block 2 - Group Identity

\begin{tabular}{lll}
\hline Variable & Survey Question & Node Strength \\
\hline V213 & I see myself as part of my local community & 0.925 \\
V215 & I see myself as part of the [regional organization] & 0.925 \\
\hline
\end{tabular}

of the variables are equal. Similarly, as Block 2 only contains one edge, no network graph was drawn, as a graph would provide no additional information.

Block 3 contains a series of questions related to what degree certain infractions can be justified depending on the circumstances or if these infractions are never justifiable. This series, shown in Table 8, asks the respondent to make moral judgements on certain situations, allowing the respondents to express either a more absolute or relative moral code (Baghramian and Carter 2019). Some respondents show greater flexibility in whether an action can be justified, while other show more stringent moral orientations, deeming that an action can never be justified regardless of the circumstances. This series includes questions on whether abortion (V204), violence against other people (V210), avoiding a fare on public transport (V199), and claiming government benefits to which you are not entitled (V198) are ever justifiable. All variables of this series that are included in the overall network appear in this block. V187 asks about

Table 8 Survey questions: Block 3 - Absolute/Relative Moral Orientation

\begin{tabular}{|c|c|c|}
\hline Variable & Survey Question & $\begin{array}{l}\text { Node } \\
\text { Strength }\end{array}$ \\
\hline V204 & $\begin{array}{l}\text { T]ell me... whether you think [abortion] can always be justified, never be justified, or } \\
\text { something in between }\end{array}$ & 9.445 \\
\hline V66 & [W]ould you be willing to fight for your country? & 8.734 \\
\hline V100 & $\begin{array}{l}\text { In the long run, hard work usually brings a better life - Hard work doesn't generally } \\
\text { bring success; it's more a matter of luck and connections }\end{array}$ & 8.699 \\
\hline V60 & $\begin{array}{l}\text { Priority for the country - first choice: A high level of economic growth; Making sure } \\
\text { this country has strong defense forces; Seeing that people have more say about how } \\
\text { things are done at their jobs and in their communities; Trying to make our cities and } \\
\text { countryside more beautiful. }\end{array}$ & 8.678 \\
\hline V182 & $\begin{array}{l}\text { To what degree are you worried about [not being able to give my children a good } \\
\text { education] }\end{array}$ & 8.622 \\
\hline V45 & When jobs are scarce, men should have more right to a job than women & 8.375 \\
\hline V39 & $\begin{array}{l}\text { Please mention[if] you would not like to have [immigrants/foreign workers] as } \\
\text { neighbors }\end{array}$ & 7.740 \\
\hline V99 & $\begin{array}{l}\text { Competition is good. It stimulates people to work hard and develop new ideas - } \\
\text { Competition is harmful. It brings out the worst in people. }\end{array}$ & 7.506 \\
\hline V148 & Do you believe in God? & 7.201 \\
\hline V187 & Under some conditions, war is necessary to obtain justice & 6.576 \\
\hline V210 & $\begin{array}{l}\text { T]ell me... whether you think [violence against other people] can always be justified, } \\
\text { never be justified, or something in between }\end{array}$ & 6.321 \\
\hline V199 & $\begin{array}{l}\text { T]ell me... whether you think [avoiding a fare on public transport] can always be } \\
\text { justified, never be justified, or something in between }\end{array}$ & 5.972 \\
\hline V41 & $\begin{array}{l}\text { Please mention[if] you would not like to have [people of a different religion] as } \\
\text { neighbors }\end{array}$ & 5.645 \\
\hline V198 & $\begin{array}{l}\text { T]ell me... whether you think [claiming government benefits to which you are not } \\
\text { entitled] can always be justified, never be justified, or something in between }\end{array}$ & 5.471 \\
\hline V128 & $\begin{array}{l}\text { Having experts, not government, make decisions according to what they think is best } \\
\text { for the country [is a very good way of governing a country] }\end{array}$ & 3.898 \\
\hline
\end{tabular}




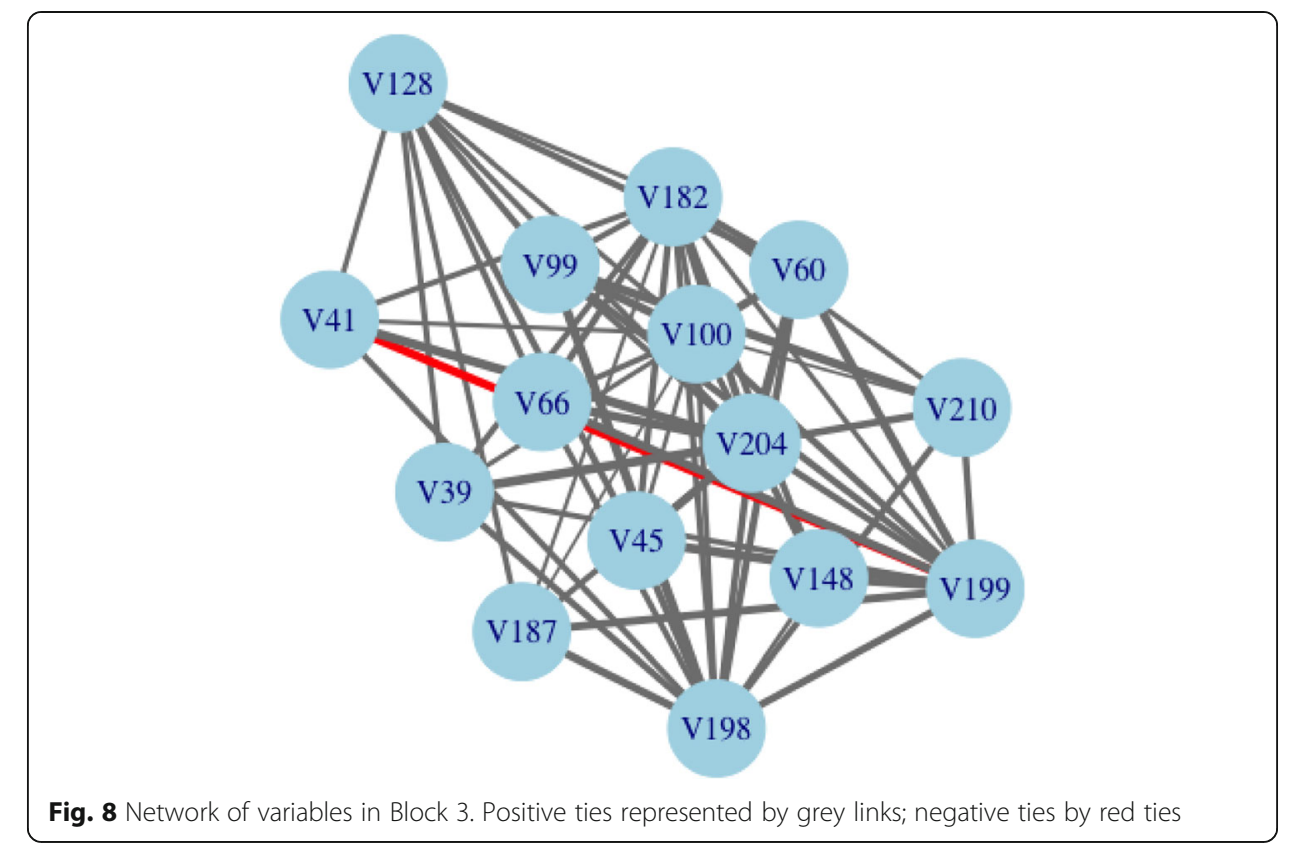

the justifiability of action on the part of a nation, whether there are times when it is necessary to go to war.

In addition to variables of the moral orientation series, the block includes one religious question, namely V148, does the respondent believe in God. The block also includes a question commonly used to measure national pride (V66). Finally, two variables related to competition and success are included in the block: V100, whether hard work usually brings about a better life or whether success is more dependent on luck and connections, and V99, the value of competition.

Block 3 also contains questions that are especially relevant to the dependent variable. The first poses a similar question as the dependent variable, asking whether men should have more right to a job than women when jobs are scarce (V45). The block also contains two questions out of a series related to prejudice and stigma. These variables ask whether the respondent would prefer not to have immigrants and foreign workers as neighbors (V39) as well as whether the respondent would not like to have people of a different religion as neighbors. Other variables in this series are included in the network, but appear in Block 6 and Block 8 .

Finally, this block contains the only negative ties between variables within the same cluster. These negative ties appear between V41, whether or not the person would not like to have people of a different religion as a neighbor, and V210 and between V41 and V199. V210, whether violence against other people is justifiable, and V199, whether avoiding a fare is justifiable, are strongly related, with an dot product of 0.867 , as seen in Appendix G. V41 and the two variables are negatively correlated, though to a fairly weak degree. The dot product between V41 and V199 is - 0.133, while the dot product between V41 and V210 is negligible at -0.002 .

Figure 8 shows the variable network in Block 3. Positive ties between variables are represented by grey edges, while negative ties are represented by red ties. Furthermore, the edge weight is represented by the width of ties, with edges of larger weights having 
Table 9 Survey questions: Block 4 - Democracy

\begin{tabular}{|c|c|c|}
\hline Variable & Survey Question & $\begin{array}{l}\text { Node } \\
\text { Strength }\end{array}$ \\
\hline V140 & How important is it for you to live in a country that is governed democratically & 3.051 \\
\hline V218 & Indicate whether you use [printed magazines] to obtain information & 2.642 \\
\hline V101 & $\begin{array}{l}\text { People can only get rich at the expense of others - Wealth can grow so there's } \\
\text { enough for everyone }\end{array}$ & 2.418 \\
\hline V55 & $\begin{array}{l}{[\mathrm{H}] \text { ow much freedom of choice and control [do] you feel you have over the way your }} \\
\text { life turns out? }\end{array}$ & 2.262 \\
\hline V97 & $\begin{array}{l}\text { Private ownership of business and industry should be increased - Government } \\
\text { ownership of business and industry should be increased }\end{array}$ & 1.345 \\
\hline V71 & It is important to this person to be rich; to have a lot of money and expensive things & 1.298 \\
\hline
\end{tabular}

greater thickness. While two negative ties appear in the network, the edge weight of the link between V41 and V210 is so low that the edge is not visible in this network.

The keyword for Block 4 is democracy, taken from the V140, the most central node in Block 4. This variable asks the respondent if it is important to them to live in a democratically governed country. The other variables included in this block are detailed in Table 9.

The other variables in the cluster all show the highest degree of similarity with the question of democracy, implying that views about importance of wealth and how it can be accumulated (V71 and V101), how much freedom of choice and control the respondent feels (V55), and government ownership of businesses (V97) are correlated with the overall importance of democracy to a person. V71, whether it is important for the respondent to be rich, is the only exception, as it shows a very small degree of greater similarity to questions about how wealth can be accumulated. Whether or not democracy is important for respondents shows a high degree of similarity to how respondents obtain information, as represented by V218.

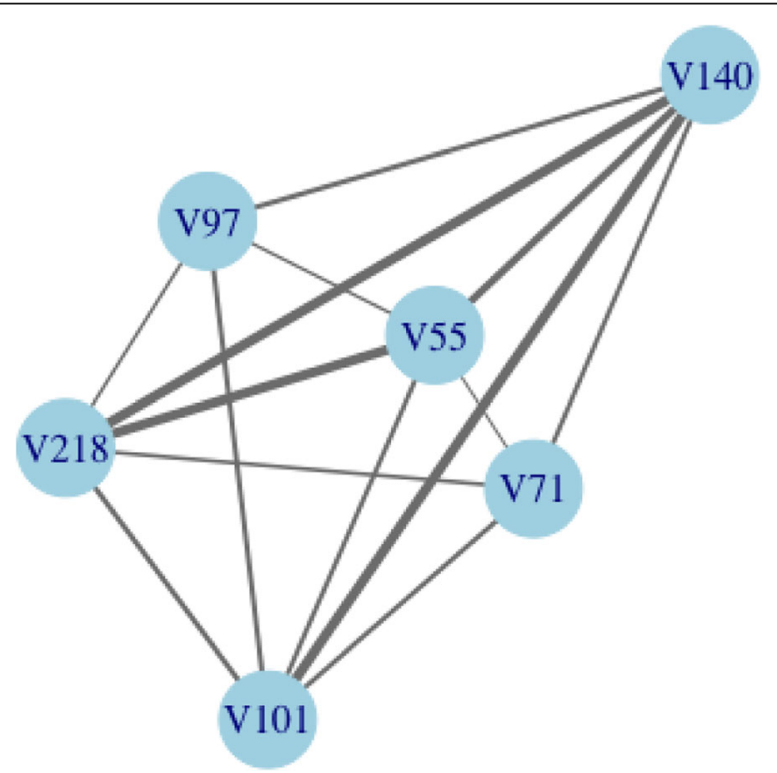

Fig. 9 Network of variables in Block 4. Positive ties represented by grey links; negative ties by red ties 
Table 10 Survey questions: Block 5 - Science and Technology

\begin{tabular}{lll}
\hline Variable & Survey Question & $\begin{array}{l}\text { Node } \\
\text { Strength }\end{array}$ \\
\hline V192 & Science and technology are making our lives healthier, easier, and more comfortable & 0.260 \\
V61 & $\begin{array}{l}\text { Priorities for the country- second choice: A high level of economic growth; Making } \\
\text { sure this country has strong defense forces; Seeing that people have more say about } \\
\text { how things are done at their jobs and in their communities; Trying to make our cities } \\
\text { and countryside more beautiful. }\end{array}$ & 0.260 \\
& & \\
\hline
\end{tabular}

As the Block 4 network shown in Fig. 9 demonstrates, the block is a clique. All variables are correlated with one another, to greater or lesser degrees as depicted by the varying edge widths.

Block 5 comprises two questions. One asks the respondents whether they agree with the statement that science and technology is improving life (V192). The second question asks what is their respondents' priority for the country (V61). This variable is part of a two-part series which asks about priorities for countries. The related variable, V60, asks respondents what their first priority for a country is, as shown in Table 10.

Block 6 contains four out of the remaining five questions in the series related to prejudice and stigma, described in Table 11. These variables ask whether the respondent would not like to live near drug addicts (V36), people with AIDS (V38), homosexuals (V40), and heavy drinkers (V42). Only one other variable is included in the block, which asks the respondent if they identify as a religious person (V147).

As Fig. 10 show, the block is a clique, and all variables are correlated with one another.

Block 7 represents the only singleton block, meaning the block comprises only one variable. This variable, V150, asks whether the basic meaning of religion is to follow norms or to do good to other people. Here, the survey the respondent to consider the meaning and value of religion for its dogmatic or practical qualities. This variable attempts to understand how religion motivates behavior. The question is shown in full in Table 12.

Detailed in Table 13, Block 8 comprises two questions, the first about religion (V151) and the second regarding stigma surrounding unmarried couples (V48). V151 asks whether the basic meaning of religion is to make sense of this life or of life after death, resembling the singleton variable of V150. In this case, the respondent is asked for the role of religion in understanding the universe and human experience, characterized as both life before and after death. Finally, this block includes the final variable on the series of prejudice and stigma, in this case towards unmarried couples living together.

Table 11 Survey questions: Block 6 - Prejudice and Stigma

\begin{tabular}{lll}
\hline Variable & Survey Question & $\begin{array}{c}\text { Node } \\
\text { Strength }\end{array}$ \\
\hline V36 & Please mention[if] you would not like to have [drug addicts] as neighbors & 3.246 \\
V38 & Please mention[if] you would not like to have [people who have AIDS] as neighbors & 3.182 \\
V40 & Please mention [if] you would not like to have [homosexuals] as neighbors & 2.941 \\
V42 & Please mention[if] you would not like to have [heavy drinkers] as neighbors & 2.863 \\
V147 & Independently of whether you attend religious services or not would you say you are & 2.269 \\
& [a religious person] & \\
\hline
\end{tabular}




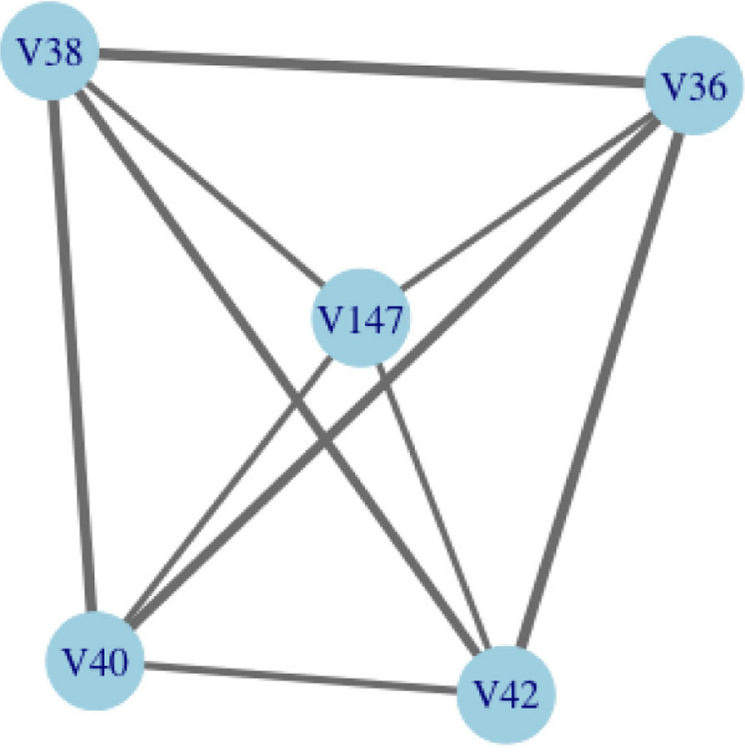

Fig. 10 Network of variables in Block 6. Positive ties represented by grey links; negative ties by red ties

Having classified each block according to the keyword, it is possible to arrange the determinants into a network graph, showing the positive or negative relationships between each determinant. This network is shown in Fig. 11.

The edges between determinants can have negative or positive signs, as shown by the red and green edges respectively. The edges have no weights.

\section{Discussion}

\section{Countries}

The nine countries selected have greater similarities than differences in their determinants of attitudes towards immigrants, as seen by the network graph and by the positive ties between all countries. The countries form a clique; each country has a positive degree of similarity to all other countries. Dividing the network into communities decreased the overall modularity of the clusters, indicating that all nodes belonged in a single cluster. The ties between some countries show higher weights, meaning they are more similar in their determinants of attitudes. Thailand and Taiwan have the strongest edge in the network, with a weight of 0.64, suggesting that these countries are very similar in their determinants. Overall, the Philippines shows the weakest node strength, meaning the sum of its all its edge weights are the smallest. For this reason, the Philippines is the most unique in its determinants in this sample. Thus, there does not appear to be any significant difference in the determinants of attitudes between countries of different development levels, migration profile, or between East Asian and Southeast Asian countries.

Table 12 Survey questions: Block 7 - Religion (and behavior)

\begin{tabular}{ll}
\hline Variable & Survey Question \\
\hline V150 & $\begin{array}{l}\text { The basic meaning of religion is to follow religious norms and ceremonies [or] to do good to other } \\
\text { people }\end{array}$
\end{tabular}


Table 13 Survey questions: Block 8 - Religion 2 (and cognition)

\begin{tabular}{lll}
\hline Variable & Survey Question & $\begin{array}{l}\text { Node } \\
\text { Strength }\end{array}$ \\
\hline V151 & $\begin{array}{l}\text { The basic meaning of religion is to make sense of life after death [or] to make sense } \\
\text { of life in this world }\end{array}$ & 0.347 \\
V43 & $\begin{array}{l}\text { Please mention[if] you would not like to have [unmarried couples living together] as } \\
\text { neighbors }\end{array}$ & 0.347 \\
\hline
\end{tabular}

Due to the size of the sample and the geographic and cultural similarities amongst the countries, the country network only shows slight variations amongst countries, with some countries being slightly more similar and some countries more unique in their determinants. Expanding the size of the sample will allow the differences amongst regions to become more apparent and provide a clearer picture of how macro-level factors affect the determinants of attitudes. However, from this country network, one can conclude that the countries in this sample are broadly similar, and that there does not appear to be significant differences between East Asian and Southeast Asian countries, developed or developing countries, or migrant senders or migrant receivers.

\section{Variables}

The findings of this study have implications for the prevailing theories of how attitudes towards immigrants form and the applicability of these theories to countries outside European and Settler countries. Perhaps the most surprising finding, education was not found to be a significant variable in determining attitudes towards immigrants for any countries in the sample, despite its strong support in the literature. Moreover, the

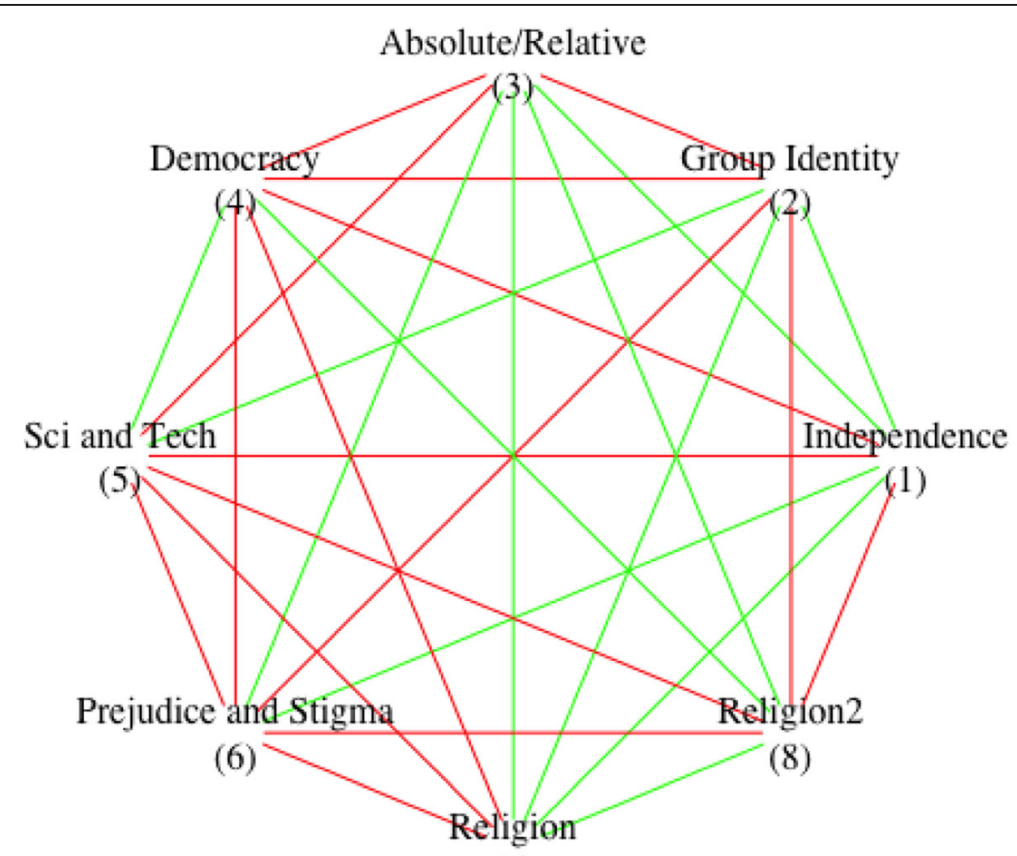

(7)

Fig. 11 Network of determinants. Green ties represent positive edges; red edges represent negative ties. Edges have no weight 
variable measuring education was automatically included in the multiple logistic regression in the pre-selection; however, for no country did education remain a significant predictor of attitudes, and thus was not included in the network analysis. This finding suggests that education, despite being a consistent predictor in Europe and Settler countries, is not as relevant in determining attitudes outside of these regions. This result echoes that of Meseguer and Kemmerling (2016), who found a limited effect of education on attitudes in Latin American countries. Exactly why education loses its predictive power outside of the West requires further investigation. This finding lends support to the theory that education correlates more closely with cultural values in European and Settler countries, rather than representing whether a respondent is likely to be in competition with immigrants (Hello et al. 2006; Davidov and Meuleman 2012). There are several possible mechanisms through which education may affect cultural values; education may foster more reflexivity and critical thinking, reducing prejudice towards other groups (Gang et al. 2013; Chandler and Tsai 2001). Or, higher education may be more diverse places, allowing for people to from more and deeper relationships with people from other groups. Or, respondents who attain higher education may be self-selected, due to income, social class, etc. While this study's sample of countries is still too small to ascertain why exactly education loses salience in Asian countries, this finding will hopefully inspire greater investigation into the different ways in which higher education functions in the economic systems of different countries as well as how the education system itself instills or correlates with other cultural values related to attitudes towards immigrants.

Unlike education, subjective social class was included in the variable network. For most countries in the sample, social class had no significant relationship with the dependent variable and, as such, its exact relationship with attitudes towards immigrants cannot be concluded. For the countries in which social class did have a significant effect, the sign of the coefficient was not consistent. In China and Singapore, respondents who identified as belonging to lower social classes were more likely to express negative attitudes towards immigrants. This result would appear to support labor market competition theories in which people of lower classes and who are in competition with immigrants for jobs would have more negative attitudes towards immigrants. However, in Thailand, respondents in higher classes were more likely to express negative attitudes towards immigrants. Social class encompasses not only income but also nonmaterial elements of prestige and social standing. Its inclusion in Block 1 (independence and social dependencies), the high degree of similarity to variables related to institutional trust, as well as the absence of income in the network of variables implies that the effect of social class on attitudes is more closely related to the social standing aspects of class than its economic dimensions. Moreover, the differing signs between countries in which class had a significant effect implies that class may have a mediating effect on attitudes, but that it depends on the overall social and economic context of the country. This study can, thus, conclude that the effect of social class appears to be more closely related to social standing rather than the material aspects of class and that the effect of class on attitudes depends on the social and economic context of a country.

Two determinants, science and technology and absolute/relative moral orientation, had not been mentioned by the literature review and warrant further research. Other determinants had been mentioned by the previous literature but show different relationships than 
had been hypothesized. The results of this study support, contradict, and expand theories related to determinants of attitudes, specifically the effects of education, social class, group identity, religion, prejudice, and anxiety on attitudes towards immigrants.

Theories of prejudice often cite social identity theory to explain how group identities affect individuals' prejudice against immigrants and minorities. Both group identities and prejudice were identified as determinants and were negatively correlated, though weakly. This finding suggests that greater attachment to one's local community or to one's regional organization was correlated with greater prejudice. However, for most countries, the relationship between group identity and negative attitudes towards immigrants was not statistically significant. Thus, while it does appear that prejudice and group identity have a negative relationship, this relationship requires further investigation due to the small sample size.

For the countries for which there was a significant relationship between group identity and negative attitudes towards immigrants, the signs of these relationships were not consistent. Respondents in Singapore who saw themselves as more closely tied with either their local community or the regional association were more likely to have positive attitudes towards immigrants, while respondents in the Philippines who felt more closely tied to both groups were more likely to have negative attitudes towards immigrants. Both forms of attachment caused respondents in the Philippines to become more negative towards immigrants, while the opposite trend emerged in Singapore. As both forms of attachment behave very similarly, it appears that the object of attachment is not nearly as important as the strength of the attachment. However, due to the inconsistency of the sign, how group identities affect attitudes towards immigrants appears to be country-specific. As these variables were significant for few countries in the sample, the exact relationship between attitudes and group identities cannot be concluded from this study.

Religion plays a diffuse role in determining attitudes towards immigrants, with variables measuring different aspects of religion having very different relationships with other variables. All four variables related to religion fell into different blocks. Overall, questions regarding religion show a negative, though very weak, correlation with stronger group identity (Block 2), democracy (Block 4), and science and technology (Block 5). In their relationships with other clusters, the major distinction between variables related to religion lies in their interaction with Block 1 (independence and social dependencies) and Block 6 (prejudice and stigma). In other words, what facet of religion is being discussed becomes salient only with regards to prejudice and stigma, and independence. Belief in God as well as whether the respondent identifies as religious does impact people's prejudices and stigmas; however, how one interprets the meaning of religion has no bearing on prejudices and stigmas. Furthermore, whether or not one believes in God does not affect whether one believes that the meaning of religion is to follow norms or to do good towards others, but is correlated with all other variables regarding religion.

Stigma and prejudice against foreign workers and people of a different religion appear to be qualitatively different from prejudice against other groups. As the previous paragraph mentions, the major causes of differentiation between variables in this series are their relationships with Block 7 and Block 8, determinants related the meaning of religion. Whereas variables in Block 6 have negative edges with Block 7 and Block 8, V41, 
which asks whether the respondent would like to have neighbors of a different religion, shows a fair amount of similarity with both; the inner products of the relationship of V41 with V150 and V151 are 0.346 and 0.228 , respectively. V39, which asks about prejudice and stigma against immigrants, has a lesser degree of dissimilarity from Block 6 , as it has a weakly negative inner product with V150 and a weakly positive inner product with V151. This pattern suggests that prejudice against people of different religions or against immigrants correlates with the person's conception of the meaning of religion. However, stigma and prejudice towards other groups, such as drug addicts, homosexuals, or people of another religion, is not correlated with the meaning of religion.

Finally, this study finds that variables related to anxiety are located in blocks related to independence and absolute/relative moral orientations, Blocks 1 and 3 respectively. In their study on the role of anxiety in information seeking about immigration, Gadarian and Albertson identified four major causes of concern: economic concerns, concerns about exploiting the social welfare system, cultural worries, and security concerns (2014). The placement of variables related to anxiety in certain blocks suggest that anxiety may operate on certain considerations to a larger extent than others. In particular, Block 3 relates to the absolute and relative moral orientation of respondents. The placement of the fear here may suggest that affronts to permissible behavior creates greater feelings of threat than other considerations. This finding helps explain the precedents of the much more stringent and punitive reactions to illegal immigration than documented immigration (Hood and Morris 1998). Variables measuring anxiety in Block 1, which measures independence as well as institutional trust and normative behavior, reinforce this interpretation. These findings suggest that fears about the cultural threat posed by immigrants' inclusion into society may have less to do with group identity and prejudice than a more rigid adherence to established, normative behavior. Interestingly, anxiety was not included in clusters regarding prejudice, but these variables measuring anxiety did show a positive correlation with the series of variables regarding prejudice and stigma in both Blocks 3 and 6. Furthermore, anxiety has a somewhat mixed relationship with group identity, with concerns about providing a good education for their children showing negative edges and other measures of anxiety showing a very low degree of similarity. These findings suggests that anxiety does have a galvanizing effect on prejudices, but that it is moderated through anxieties related to permissible behavior.

\section{Conclusion}

This study has found that overall, the countries in the sample represent a cohesive group, showing greater similarities than differences, despite differences in development level and migration profile. The one-mode projection of the variables produced eight determinants of attitudes: independence, group identity, absolute/relative moral orientation, democracy, science and technology, prejudice and stigma, and two determinants related to the meaning of religion. Some of these determinants had not been previously identified by research, including science and technology and absolute/relative moral orientation.

The relationships between variables, as well as what variables were included in each cluster, have complicated and expanded the previous research on determinants. Several variables which were assumed to be relatively consistent in their predictive power were found to be insignificant, as in the case of education, or inconsistent in the direction of 
the effects, as in the case of class. In particular, the absence of education as a significant variable stresses the need for greater research into countries outside of Europe and the Settler countries. This finding complicates the proposed relationship between education and labor market competition and reinforces the hypothesis that higher education in European and Settler countries correlates with cultural values that liberalize attitudes towards immigrants. However, before concluding that education's effect on attitudes occurs because the curriculum is inspiring greater critical thinking in its students or through self-selection, it is necessary to evaluate how education mediates other socioeconomic aspects of life. The meaning of education, as a class signifier, a determinant of labor market outcomes, and its relationship with, depends on the country context and could conceivably be related to macro-level factors related to inequality or social mobility. Despite the abundance of studies which include education, the exact relationship between education and attitudes towards immigrants requires further research. Equally, the effect of class appears to be country-specific, as seen by the differing signs of its coefficients. Future research would do well to investigate under what conditions members of lower classes feel antagonism towards immigrants, as in the case of China and Singapore, rather than solidarity with immigrants, as in the case of Thailand.

Supporting social identity theory, stronger group identities were found to be correlated with prejudice; though due to the small sample size, this trend cannot be confirmed. Interestingly, the object of attachment, whether it was one's local community or a regional organization, appeared to be less significant than the strength of the attachment, a finding that should be confirmed and expanded upon in future research.

In this study, prejudice and religion appeared to be interrelated, as variables in these series were differentiated due to their relationship with variables related to religion. In particular, variables related to prejudice against immigrants/foreign workers and people of different religions were separated from variables related to prejudice against groups, like drug users, people suffering from AIDS, and so on, because of their relationships with variables related to the meaning of religion. Thus, mentioning and discussing religion is not necessarily in conflict with discussion regarding prejudice; in fact, the two can be mutually reinforcing when speakers carefully choose which facet of religion to discuss. Stressing religious identification and belief in God may activate prejudice, while discussions of the meaning of religion may mitigate it.

Finally, anxiety, which had been identified by previous research as a salient determinants, is found to operate specifically on people's expectations of normative social behavior, as seen by its inclusion in Block 3 (absolute/moral orientation) and Block 1 (independence and social dependencies). As anxiety appears to be correlated with expectations of how others should behave and whether one has a greater or lesser ability to accept non-normative behavior, media likely plays a large role in moderating or exacerbating fears. Policymakers and others may find depictions of the mundane social expectations and rules of immigrant and autochthonous populations to be fruitful pathways to quell fears related to immigration.

While the relationships of these determinants and the macro-level factors that may be underlying them cannot be concluded in all cases, this study hopes to inspire greater research into these understudied areas and to expand the field. Research should reflect the changing nature of migration, including expanding into understudied regions, and should employ new techniques, such as network science, where applicable. While 
methodological difficulties exist, identifying generalizable variables and the macro-level economic and social conditions that mediate them can only enrich understanding of when and why a society accepts or rejects newcomers.

\section{Supplementary information}

Supplementary information accompanies this paper at https://doi.org/10.1007/s41109-020-00315-W.

Additional file 1: Appendix A. Spearman's correlation - Variables with high correlation. Appendix B. Chisquared test of independence - Retained variables. Appendix C. Linear regression of continuous variables. Appendix D. Multiple logistic regression results. Appendix E. Bipartite network weighted adjacency matrix. Appendix F. Normalized weighted adjacency matrix: country network. Appendix G. Normalized weighted adjacency matrix: variable network.

\section{Abbreviations}

ATIl: Attitudes towards immigrants and immigration; CAN: Causal Attitude Network; ESS: European Social Survey; ISSP: International Social Survey Programme; LAPOP: Latin American Public Opinion Project; UN DESA: United Nations, Department of Economic and Social Affairs; WVS: World Values Survey

\section{Acknowledgements}

We are grateful to D. lalnazov and M. Deroche for their insightful comments and encouragement.

\section{Authors' contributions}

RKK provided key contributions in performing the survey of the related work, the regression analysis, and network analysis. YI supervised the project and conceptualized the bipartite graph expression of the regression analysis. All authors read and approved the final manuscript.

\section{Funding}

The present study was supported by the Ministry of Education, Culture, Sports, Science and Technology (MEXT), Grants-in-Aid for Scientific Research (B), Grant No. 17KT0034 (2007-2019).

\section{Availability of data and materials}

The datasets analyzed during the current study are available in the World Values Survey repository (Ingelhart et al. 2014), the UN DESA website (UN DESA 2013), and the World Bank website (World Bank 2019).

\section{Competing interests}

None.

Received: 9 April 2020 Accepted: 3 September 2020

Published online: 02 November 2020

\section{References}

Agudelo-Suárez A, Gil-Gonzalez D, Ronda-Pérez E, Porthe V, Paramio-Perez G, García AM, Garí A (2009) Discrimination, work and health in immigrant populations in Spain. Soc Sci Med 68(10):1866-1874

Baghramian M, Carter JA "Relativism", The Stanford Encyclopedia of Philosophy (Winter 2019 Edition)s, Edward N. Zalta https:// plato.stanford.edu/archives/win2019/entries/relativism/

Batagelj V, Mrvar A (1998) Pajek-program for large network analysis. Connections 21(2):47-57

Blondel VD, Guillaume JL, Lambiotte R, Lefebvre E (2008) Fast unfolding of communities in large networks. J Stat Mech 2008(10):P10008

Brannon LA, Tagler MJ, Eagly AH (2007) The moderating role of attitude strength in selective exposure to information. J Exp Soc Psychol 43(4):611-617

Burns P, Gimpel JG (2000) Economic insecurity, prejudicial stereotypes, and public opinion on immigration policy. Polit Sci Q 115(2):201-225

Campbell AL, Wong C, Citrin J (2006) "Racial threat", partisan climate, and direct democracy: Contextual effects in three California initiatives. Polit Behav 28(2):129

Card D, Dustmann C, Preston I (2005) Understanding attitudes to immigration: the migration and minority module of the first European social survey

Carlsson M, Eriksson S (2017) Do attitudes expressed in surveys predict ethnic discrimination? Ethn Racial Stud 40(10):17391757

Castles S (2010) Understanding global migration: a social transformation perspective. J Ethn Migr Stud 36(10):1565-1586

Ceobanu AM, Escandell X (2010) Comparative analyses of public attitudes toward immigrants and immigration using multinational survey data: a review of theories and research. Annu Rev Sociol 36(1):309-328

Chandler CR, Tsai YM (2001) Social factors influencing immigration attitudes: an analysis of data from the general social survey. Soc Sci J 38(2):177-188

Citrin J, Green DP, Muste C, Wong C (1997) Public opinion toward immigration reform: the role of economic motivations. J Polit 59(3):858-881

Coenders M, Lubbers M, Scheepers P, Verkuyten M (2008) More than two decades of changing ethnic attitudes in the Netherlands. J Soc Issues 64(2):269-285 
Coenders M, Scheepers P (2003) The effect of education on nationalism and ethnic exclusionism: an international comparison. Polit Psychol 24(2):313-343

Coenders MTA, Lubbers M, Scheepers PLH (2005) Majorities' attitudes towards minorities in European Union member states: results from the standard Eurobarometer 1997-2000-2003

Coenders MTA, Scheepers PLH (2004) Associations between nationalist attitudes and exclusionist reactions in 22 countries. In: Nationalism and exclusion of migrants: Cross-national comparisons, pp 187-208

Cooray A, Marfouk A, Nazir M (2018) Public opinion and immigration: who Favours employment discrimination against immigrants? Int Migr 56(6):5-23 https://onlinelibrary.wiley.com/doi/pdf/10.1111/imig.12457

Crano WD, Prislin R (2006) Attitudes and persuasion. Annu Rev Psychol 57:345-374

Dalege J, Borsboom D, van Harreveld F, van den Berg H, Conner M, van der Maas HL (2016) Toward a formalized account of attitudes: the causal attitude network (CAN) model. Psychol Rev 123(1):2

Davidov E, Meuleman B (2012) Explaining attitudes towards immigration policies in European countries: the role of human values. J Ethn Migr Stud 38(5):757-775

Dennison J, Geddes A (2019) A rising tide? The salience of immigration and the rise of anti-immigration political parties in Western Europe. Polit Q 90(1):107-116

Doreian P, Mrvar A (2009) Partitioning signed social networks. Soc Networks 31(1):1-11

Dustmann C, Preston I (2007) Racial and economic factors in attitudes to immigration. B E J Econ Anal Policy 7:62

Esses VM, Jackson LM, Armstrong TL (1998) Intergroup competition and attitudes toward immigrants and immigration: an instrumental model of group conflict. J Soc Issues 54(4):699-724

Facchini G, Mayda AM (2008) From individual attitudes towards migrants to migration policy outcomes: theory and evidence. Econ Policy 23(56):652-713

Facchini G, Mayda AM (2009) Does the welfare state affect individual attitudes toward immigrants? Evidence across countries. Rev Econ Stat 91:295-314

Fazio RH (1986) How do attitudes guide behavior. Handb Motiv Cogn 1:204-243

Fazio RH (1990) Multiple Processes by Which Attitudes Guide Behavior: The MODE Model as an Integrative Framework. In: Zanner M (ed) Advances in Experimental Social Psychology. Academic Press, New York

Festinger, L (1957) A theory of cognitive dissonance (Vol. 2). Stanford university press

Freeman GP, Hansen R, Leal DL (eds) (2013) Immigration and public opinion in liberal democracies, vol 52. Routledge, New York

Frey D (1986) Recent research on selective exposure to information. In: Advances in experimental social psychology, vol 19. Academic press, Tokyo, pp 41-80

Gadarian SK, Albertson B (2014) Anxiety, immigration, and the search for information. Polit Psychol 35(2):133-164

Gang IN, Rivera-Batiz FL, Yun M-S (2013) Economic strain, education and attitudes towards foreigners in the European Union. Rev Int Econ 21(2):177-190

Gijsberts M, Hagendoorn L (2017) Nationalism and exclusion of migrants: Cross-national comparisons. Routledge, London

Gorodzeisky A, Semyonov M (2009) Terms of exclusion: public views towards admission and allocation of rights to immigrants in European countries. Ethn Racial Stud 32(3):401-423

Hainmueller J, Hangartner D (2013) Who gets a Swiss passport? A natural experiment in immigrant discrimination. Am Polit Sci Rev 107(1):159-187

Hainmueller J, Hiscox MJ (2007) Educated preferences: explaining attitudes toward immigration in Europe. Int Organ 61(2):399-442 Hainmueller J, Hopkins DJ (2014) Public attitudes toward immigration. Annu Rev Polit Sci 17:225-249

Harary F, Norman RZ, Cartwright D (1965) Structural models: an introduction to the theory of directed graphs. Wiley, New York

Hart W, Albarracín D, Eagly AH, Brechan I, Lindberg MJ, Merrill L (2009) Feeling validated versus being correct: a meta-analysis of selective exposure to information. Psychol Bull 135(4):555

Hello E, Scheepers P, Gijsberts M (2002) Education and ethnic prejudice in Europe: explanations for cross-national variances in the educational effect on ethnic prejudice. Scand J Educ Res 46(1):5-24

Hello E, Scheepers P, Sleegers P (2006) Why the more educated are less inclined to keep ethnic distance: an empirical test of four explanations. Ethn Racial Stud 29:959-985 https://doi.org/10.1080/01419870600814015

Hjerm M (2007) Do numbers really count? Group threat theory revisited. J Ethn Migr Stud 33(8):1253-1275

Hood MV, Morris IL (1998) Give us your tired, your poor,... but make sure they have a green card: The effects of documented and undocumented migrant context on Anglo opinion toward immigration. Polit Behav 20(1):1-15

Inglehart R, Haerpfer C, Moreno A, Welzel C, Kizilova K, Diez-Medrano J, Lagos M, Norris P, Ponarin E, Puranen B et al (eds) (2014) World values survey: round six - country-pooled Datafile version: www.worldvaluessurvey.org/ WVSDocumentationWV6.jsp. JD Systems Institute, Madrid

Jackson JS, Brown KT, Brown TN, Marks B (2001) Contemporary immigration policy orientations among dominant-group members in Western Europe. J Soc Issues 57(3):431-456

Kehrberg JE (2007) Public opinion on immigration in Western Europe: Economics, tolerance, and exposure. Comp Eur Polit, 5(3):264-281

Kessler AE, Freeman GP (2005) Public opinion in the EU on immigration from outside the community. J Common Market Stud 43(4):825-850

Kinder DR, Kam CD (2010) Us against them: ethnocentric foundations of American opinion. University of Chicago Press, Chicago

Knabe A, Rätzel S, Thomsen SL (2013) Right-wing extremism and the well-being of immigrants. Kyklos 66(4):567-590

Knoll BR, Redlawsk DP, Sanborn H (2011) Framing labels and immigration policy attitudes in the lowacaucuses: trying to outTancredo Tancredo. Polit Behav 33:433-54.

Kunovich RM (2009) The sources and consequences of national identification. Am Sociol Rev 74(4):573-593

Lahav G (2004) Public opinion toward immigration in the European Union: does it matter? Comp Poll Stud 37(10):1151-1183

Lancee B, Sarrasin O (2015) Educated preferences or selection effects? A longitudinal analysis of the impact of educational attainment on attitudes towards immigrants. Eur Sociol Rev 31(4):490-501

Lawrence D (2011) Immigration attitudes in Latin America: culture, economics, and the catholic church. Lat Am 55(4):143-170

Malchow-Møller N, Munch JR, Schroll S, Skaksen JR (2009) Explaining cross-country differences in attitudes towards immigration in the EU-15. Soc Indic Res 91(3):371-390 
Mayda AM (2006) Who is against immigration? A cross-country investigation of individual attitudes toward immigrants. Rev Econ Stat 88(3):510-530

McLaren LM (2001) Immigration and the new politics of inclusion and exclusion in the European Union: the effect of elites and the EU on individual-level opinions regarding European and non-European immigrants. Eur J Polit Res 39(1):81-108

McLaren LM (2003) Anti-immigrant prejudice in Europe: Contact, threat perception, and preferences for the exclusion of migrants. Soc Forces 81(3):909-936

Meseguer C, Kemmerling A (2016) What do you fear? Anti-Immigrant Sentiment in Latin America. International Migration Review

Meuleman B, Davidov E, Billiet J (2009) Changing attitudes toward immigration in Europe, 2002-2007: a dynamic group conflict theory approach. Soc Sci Res 38(2):352-365

Monroe BM, Read SJ (2008) A general connectionist model of attitude structure and change: the ACS (attitudes as constraint satisfaction) model. Psychol Rev 115(3):733

Mummendey A, Klink A, Brown R (2001) Nationalism and patriotism: national identification and out-group rejection. Br J Soc Psychol 40(2):159-172

Newman BJ, Hartman TK, Taber CS (2014) Social dominance and the cultural politics of immigration. Pol Psychol 35(2):165186

O'Rourke KH, Sinnott R (2006) The determinants of individual attitudes towards immigration. Eur J Pol Econ 22(4):838-861

Peduzzi P, Concato J, Kemper E, Holford TR, Feinstein AR (1996) A simulation study of the number of events per variable in logistic regression analysis. J Clin Epidemiol 49(12):1373-1379

Penninx R (2007) Integration processes of migrants: research findings and policy challenges. Migracijske i etničke teme 23(12):7-32

Petty RE, Tormala ZL, Brinol P, Jarvis WBG (2006) Implicit ambivalence from attitude change: an exploration of the PAST model. J Pers Soc Psychol 90(1):21

Scheepers P, Gijsberts M, Hello E (2002) Religiosity and prejudice against ethnic minorities in Europe: cross-national tests on a controversial relationship. Rev Relig Res 43:242-265

Schlicht-Schmälzle R, Chykina V, Schmälzle R (2018) An attitude network analysis of post-national citizenship identities. PLoS One 13(12):e0208241

Schlueter E, Wagner U (2008) Regional differences matter. Int J Comp Sociol 49(2-3):153-173

Semyonov M, Raijman R, Gorodzeisky A (2006) The rise of anti-foreigner sentiment in European societies, 1988-2000. Am Sociol Rev 71(3):426-449

Semyonov M, Raijman R, Gorodzeisky A (2008) Foreigners' impact on European societies: public views and perceptions in a cross-national comparative perspective. Int J Comp Sociol 49(1):5-29

Sides J, Citrin J (2007) European opinion about immigration: the role of identities, interests and information. Br J Polit Sci 37(3):477-504

Smith ER, Mackie DM, Claypool HM (2015) Social psychology. Psychology Press, New York

Sniderman PM, Peri P, de Figueiredo Jr RJ, Piazza T (2000) The outsider: prejudice and politics in Italy. Princeton University Press, Princeton

Steyerberg EW, Eijkemans MJ, Habbema JDFP (1999) Stepwise selection in small data sets: a simulation study of bias in logistic regression analysis. J Clin Epidemiol 52(10):935-942

The World Bank (2019) Population, total [Data file]. https://data.worldbank.org/indicator/sp.pop.totl

United Nations, Department of Economic and Social Affairs, Population Division (2013) International Migrant Stock 2013 - By destination and origin [Data file]. https:/www.un.org/en/development/desa/population/migration/data/estimates2/ estimatesorigin.asp

United Nations, Department of Economic and Social Affairs, Population Division. (2017). The International Migration Report. https://www.un.org/en/development/desa/population/migration/publications/migrationreport/migreport.asp

Van Overwalle F, Siebler F (2005) A connectionist model of attitude formation and change. Personal Soc Psychol Rev 9(3): $231-274$

Wilkes R, Guppy N, Farris L (2007) Comment on Semyonov, Raijman, and Gorodzeisky, ASR, June 2006: right-wing parties and anti-foreigner sentiment in Europe. Am Sociol Rev 72(5):831-840

Wilson TD, Lindsey S, Schooler TY (2000) A model of dual attitudes. Psychol Rev 107(1):101

WVS Database | Who We Are (2020) WVS Database. www.worldvaluessurvey.org/WVSContents.jsp

\section{Publisher's Note}

Springer Nature remains neutral with regard to jurisdictional claims in published maps and institutional affiliations. 